\title{
The Single-Channel SSVEP-based Biometric Approach Using RNN-based Deep Models: A Systematic Comparison
}

Kutlucan Gorur ( $\sim$ kgorur@bandirma.edu.tr )

Bandirma Onyedi Eylul Universitesi

\section{Beyza ERASLAN}

Bandirma Onyedi Eylul Universitesi

\section{Research Article}

Keywords: Biometric, SSVEP, Single-Channel, Recurrent Neural Networks, Discrete Wavelet Transform

Posted Date: March 1st, 2022

DOI: https://doi.org/10.21203/rs.3.rs-1304515/v1

License: (c) (i) This work is licensed under a Creative Commons Attribution 4.0 International License.

Read Full License 


\title{
The Single-Channel SSVEP-based Biometric Approach Using RNN-based Deep Models: A Systematic Comparison
}

\author{
Kutlucan GORUR ${ }^{1,}{ }^{*}$, Beyza ERASLAN ${ }^{2}$ \\ 1,2 Electrical and Electronics Engineering, Bandirma Onyedi Eylul University, Balikesir 10200, \\ Turkey \\ Corresponding Author Email: *kgorur@bandirma.edu.tr
}

\begin{abstract}
Biometric technology is the branch of science dealing with identification and verification of individuals, focusing on physiological and behavioral traits. These traits can be reliable, permanent, unique, and capable of distinguishing one person from others. Fingerprints, the iris, and the face are conventionally used in biometric techniques involving ID cards and passwords. However, due to security threats and fraud, these static textures and approaches can be abused. Consequently, biometric studies based on electroencephalography (EEG) have received increasing attention because each individual has a dynamic and unique pattern. However, classic EEG-based biometrics have significant deficiencies, including noise-prone signals, gel-based electrodes, and the need for multi-training/multi-channel acquisition and high mental effort. In contrast, steady-state visually evoked potential (SSVEP)-based biometrics have the important advantages of low signal-to-noise ratio and untrained usage. The SSVEP signals also embrace a number of other advantages. First, the occipital lobe is the only suitable region for collecting SSVEP signals without obstruction. Second, because elicited dynamic brain responses are a natural subconscious activity, the omnipresent SSVEP signals are the most frequently emitted signals. Anyone can continuously look into flickering lights having distinct frequencies, such as cell phone flashes, without extra physical or mental effort. Few studies involving multi-channel/multi-trial SSVEP-based biometric research are available in the current literature. Moreover, there is a lack of research comparing them to the single-
\end{abstract}


channel single-trial SSVEP-based biometric approach using dry electrode-implemented recurrent neural network (RNN)-based deep learning models. To the best of our knowledge, no prior work has proposed such a biometric comparison of the RNN-based deep learning models and different sized time-series data compiled over raw and discrete wavelet transform (DWT)-based SSVEP signals. By achieving up to $100 \%$ accuracy using 11 individuals, the biometric recognition results are promising. This single-channel SSVEP-based biometric approach using RNN-based deep learning models may offer low-cost, user-friendly, and reliable individual identification authentication, leading to significant application domains.

Keywords: Biometric, SSVEP, Single-Channel, Recurrent Neural Networks, Discrete Wavelet Transform

\section{Introduction}

In modern life, with the use of passports, credit cards, laptops, and mobile phones, the demand is increasing for automatic methods of identifying people via anatomical characteristics or behavioral features [1, 2]. Biometric person recognition technologies have become an important research area in recent years and have earned an important place in many fields of application [3]. As security and privacy become more and more important, authentication systems based on biological traits are actively and widely used, even in daily life [4]. Unlike fingerprints, facial recognition, palm veins, or other external biological features, brainwave patterns can create an internal representation of traits that define a specific person [4]. However, each physiological and behavioral trait has advantages and drawbacks [2]. In order to advance an effective electroencephalographic (EEG) biometric approach, the imperative and fundamental approach is to form an EEG pattern that is consistent over time and that is a unique feature among individuals [4]. Moreover, this kind of EEG-based biometric model should have high inter-subject variability and low intra-subject variability [4]. Among 
researchers, the identification of a person by employing EEG signals is an attractive concept. However, the nature of producing targeted EEGs involves some important challenges and complexities in sensing EEG signals [5, 6]. First of all, EEG-based human-machine interfaces require high concentration, continuous mental effort, and training [6]. Moreover, channel numbers/multi-channel recordings limit the use of EEG signals in real-world biometric applications, especially in wearable technologies [5-7]. At this point, steady-state visually evoked potentials (SSVEPs), when evoked in low-frequency signals, can provide high intersubject variability and low intra-subject variability with a single-channel approach $[2,8]$.

The visual evoked potential (VEP) signals form the most widespread brain pattern implemented in clinical and cognitive neuroscience. These signals are generally elicited by image stimulation [9]. Moreover, if the subject gazes at a flickering light of a specific frequency, the brain activity response can be acquired via electrodes placed on the occipital lobe. A clearly detectable and measurable signal occurs after the stimulus. After the transient visual, this stimulus can produce a stable VEP, known as the steady-state VEP (SSVEP), which occurs at the frequency of the stimulus and its multiples. The SSVEP has a robust characteristic against artifacts, including eye blinking and body movements, and exhibits a high signal-tonoise ratio [10]. Moreover, the SSVEP can provide a high information transfer rate (ITR), with no training required for first-time usage [10-12]. Hence, these features can make the SSVEP a highly potential candidate for structuring real-time usage in human-computer interfaces consisting of biometric applications [10,11]. A great number of EEG-based and VEP-based biometric studies dealing with multi-trial and multi-channel recordings can be found in the literature [13-19]. However, few studies are based on the single-channel dry-electrode SSVEP approach with single-trial person identification $[9,20]$. Furthermore, to date, no investigation has been carried out systematically comparing the different types of recurrent neural network 
(RNN)-based deep architecture for the single-channel and single-trial SSVEP-based biometric approach, including the discrete wavelet transform (DWT).

Min et al. studied SSVEP-based identification using the neurodynamics of the elicited brain responses and electro-ocular activity (Electrooculogram/EOG) while paying particular attention to the Korean letters. These evoked potentials and EOG activity were decoded using EEG channels over the F1 (for vertical EOG activity) and F7 and F8 (for horizontal EOG activity). Row segments $(5,6$, and $7 \mathrm{~Hz})$ and column segments $(5.5,6.5$, and $7.5 \mathrm{~Hz})$ were induced in a grid-shaped line array to construct a Korean letter. According to the study, 20 persons were classified using Support Vector Machine (SVM) with an accuracy score of $98.60 \%$ [21]. In another study, four flickering frequencies $(6,12,18$, and $24 \mathrm{~Hz})$ were implemented to stimulate brain responses via nine green light-emitting diodes (LEDs). A total of 19 EEG channel recordings were obtained the scalps of the subjects. Hence, using between 5 and 19 channels, 25 individuals were recognized within an accuracy range of $64.40-100 \%$. MelFrequency Cepstral Coefficients (MFCCs) and Autoregressive Reflection (AR) coefficients were determined as discriminative features [22]. Another study was conducted to structure biometric authentication using deep learning (Convolutional Neural Network/CNN and Long Short Term Memory/LSTM) and traditional classifiers. It was noted that CNN and LSTM provided the highest average accuracies, $69.03 \%$ and $66.89 \%$, respectively, for 11 persons. In the study, a leave-one-subject-out cross-validation (LOSO-CV) was carried out to process the SSVEP signals. In the experiment, 256 EEG channels and 5 non-overlapping flickering frequencies were used [23].

Deep learning is a state-of-the-art machine learning algorithm that powers many areas of research studies and modern life applications (e.g., image processing, pattern recognition, and 
brain-computer interfaces/BCls) $[24,25]$. In the present research study, 11 participants were used for personal authentication performance based on the single-channel SSVEP approach via deep learning algorithms (Long Short-Term Memory/LSTM, Bidirectional Long Short-Term Memory/BiLSTM, and Gated Recurrent Units/GRU) with DWT. Recurrent Neural Network (RNN)-based deep learning algorithms, including LSTM, BiLSTM, and GRU, are specialized and recognize the pattern in the uncovering of temporal dependencies such as biological timeseries data [26-28]. The EEG signals consisted of waves of various frequencies. Thus, the DWT was able to break them into frequency bands to assist the deep learning algorithms during the recognition of SSVEP patterns for each individual's signal [29]. In this study, the DWT was employed to separate the sub-bands of the recorded signals. The results were processed by the RNN-based deep learning algorithms over the raw signals, and the DWT-based signals showed very promising biometric classification, offering a single-channel SSVEP biometric approach using dry-sensor technology [8]. Unlike traditional $\mathrm{BCl}$ helmets, dry-sensor technology can greatly improve wearable comfort and therefore, can be used quite successfully in biometric person recognition, as one of the next-generation Internet of Things (loT) applications $[2,7,8]$.

The introduction has presented the dataset, methods, and performance metrics. The rest of the article is structured as follows. The Results section presents the biometric recognition outcomes for the deep architecture over the raw and sub-band signals. An estimation of all outcomes and a literature overview are found in the Discussion section. Finally, the Conclusion section defines precisely the aim and outcomes of the study and its contributions to the existing literature. 


\section{Materials and Methods}

\subsection{Experimental Setup and Mainframe of the Research}

Security concerns against the threats and fraud, conventional biometric techniques, including passwords or ID cards, can be abused and sometimes can be easily accessed by imposters [2]. However, biometric recognition approaches are able to provide more robustness, high accuracy performances, and hard to spoof features [2]. Moreover, easy-to-use and wearable characteristics are also defined by single-channel and dry-electrode recordings [5-8]. In this study, single-channel SSVEP biometric approach was offered to provide reliable, highly accurate, and easy-to-use for individuals using deep learning algorithms.

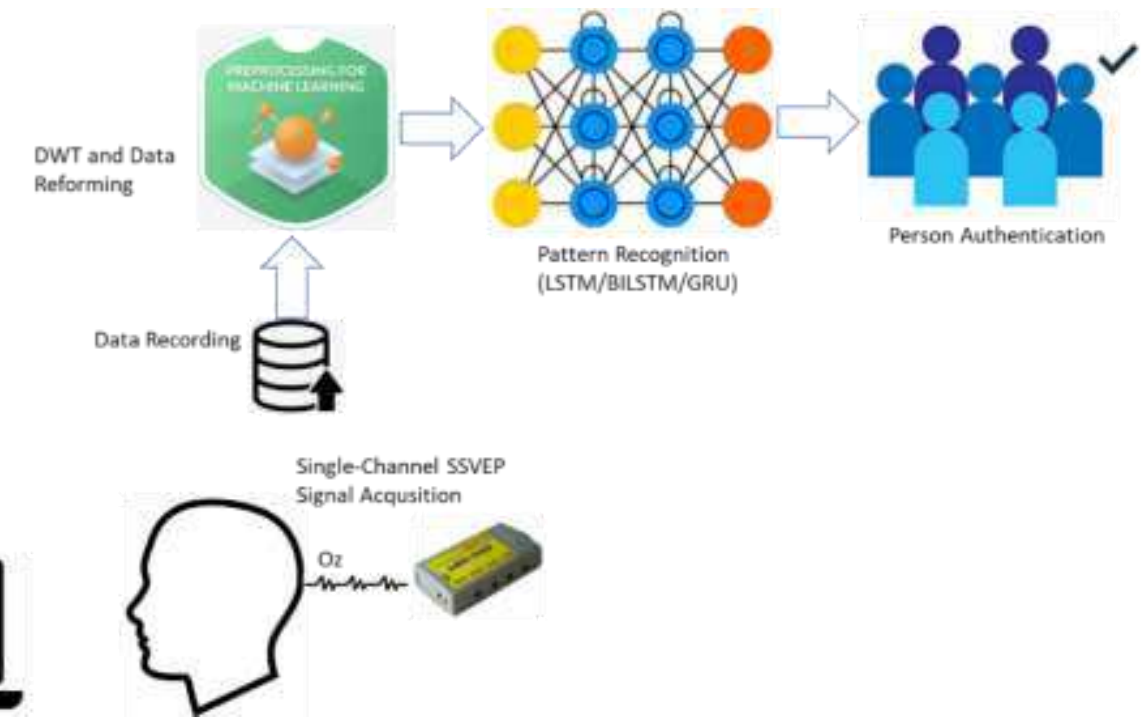

Figure 1 Single-channel dry-sensor SSVEP-based biometric approach using RNN-based deep learning algorithms over the different flicker frequencies

Steady-State Visual Evoked Potentials (SSVEP) are defined as brain signals produced in the visual cortex area while focusing on a flickering light source emitted at a specific frequency [8]. In this study, 4 frequency signals $(8.57,10,12$, and $15 \mathrm{~Hz}$ ) were reported to stimulate the brain activity for 16 seconds in collecting the dataset over the electrode of the Occipital area $(\mathrm{Oz})$. The other electrode lead of the single-channel was located on the Frontal Parietal area (Fpz). The signals of the single differential channel were noted to be sampled at $256 \mathrm{~Hz}$. These two active dry electrode pairs ( $\mathrm{CH}$ - and $\mathrm{CH}+$ ), embedded on the Olimex EEG acquisition device for a single-channel recording, were linked to the 10-bit analog-digital converter (ADC) for sampling before storing the data in the Raspberry Pi 3 minicomputer. Furthermore, a passive reference electrode was noted to be positioned on the earlobe. The 15.6-inch laptop screen 
was used for the stimulating platform. Visual stimuli have been reported to consist of four alternating black and white $80 \times 80$ pixel squares on the black background with $60 \mathrm{~Hz}$ refreshing rate of the monitor. The dataset is named "A dataset of EEG signals from a singlechannel SSVEP-based brain-computer interface" and can be accessed over this reference [8]. The bands of EEG signal and stimuli frequencies are presented in Table 1.

Table 1 EEG ranges and stimuli frequencies

\begin{tabular}{lll}
\hline \multicolumn{1}{c}{ Frequency Ranges $(\mathbf{H z})$} & \multicolumn{1}{c}{ Frequency Bands } & \multicolumn{1}{c}{$\begin{array}{c}\text { Stimulation and Observed } \\
\text { Frequencies }\end{array}$} \\
\hline $0-4$ & Delta & - \\
$4-8$ & Theta & - \\
$8-12$ & Alpha & $8.57(\mathrm{~F} 1), 10(\mathrm{~F} 2)$ \\
$12-30$ & Beta & $12(\mathrm{~F} 3), 15(\mathrm{~F} 4)$ \\
$>30$ & Gamma & - \\
\hline
\end{tabular}

Figure 1 shows the mainframe of the research and Figure 2 represents the components for the data acquisition process.
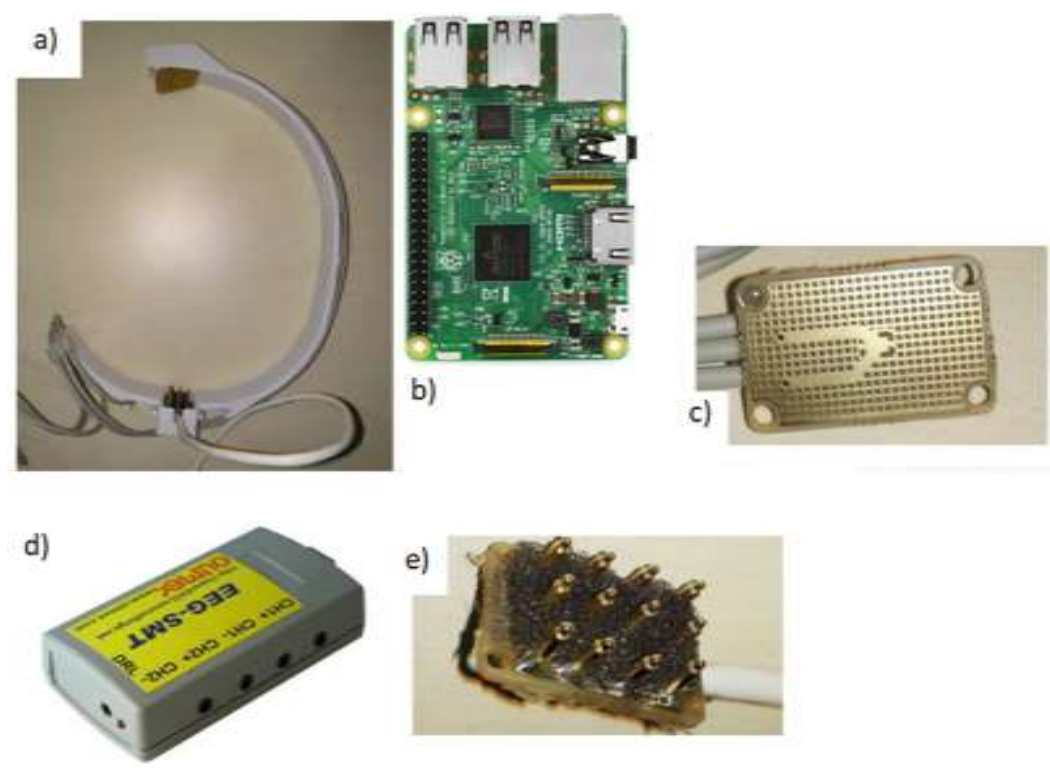


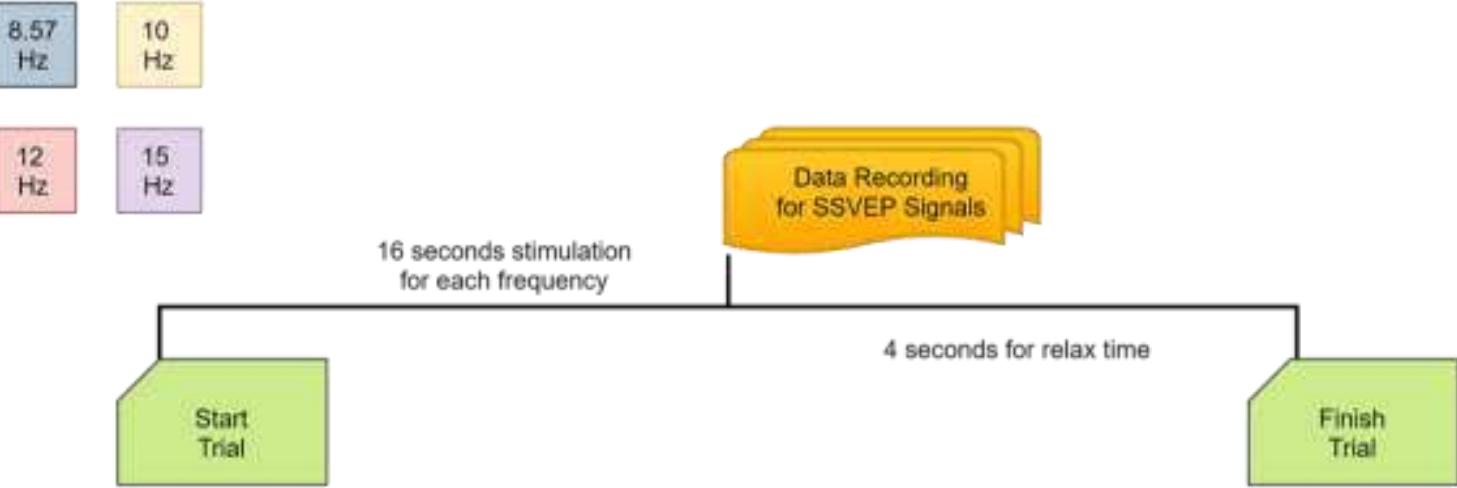

Figure 2 a) Headset b) Raspberry Pi 3 single-board computer c) FPz electrode d) Olimex EEG acquisition device e) $\mathrm{Oz}$ electrode [8] (upper) Experimental setup for trials during the stimulation process (lower)

\subsubsection{Data Preparation}

The single-channel recorded SSVEP signals for each stimulated frequency are obtained in a total of 4096 samples during 16 seconds. These signals are reshaped to merge the frequencies side by side to be recognized better via deep machine learning algorithms for 11 person biometric authentication task.

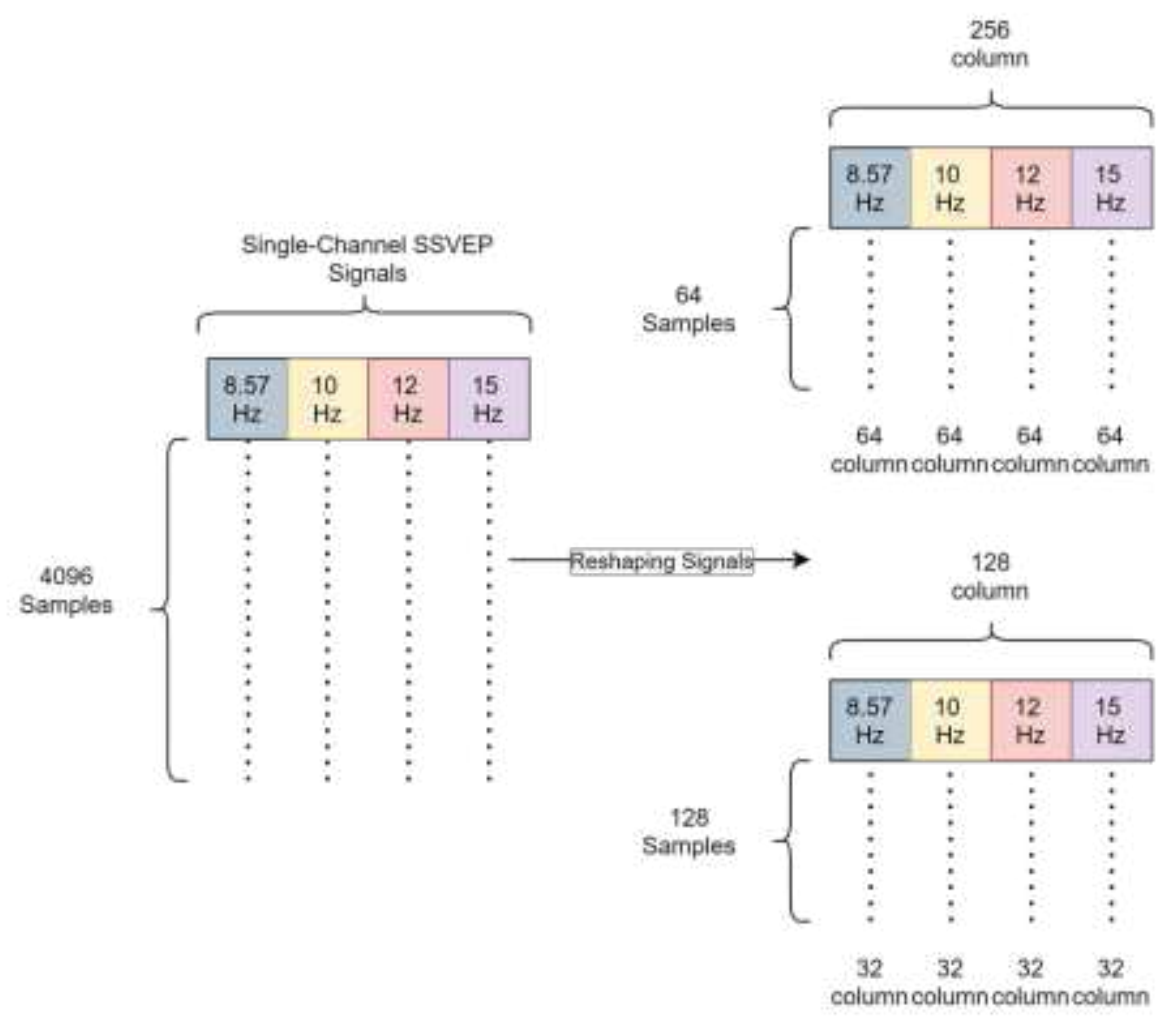

Figure 3 Resizing process for single-channel dry-sensor SSVEP signals for each person to obtain a better feature vector 


\subsection{Discrete Wavelet Transform}

The EEG signal is constructed in rhythmic activity, creating different frequency bands. These various frequencies can be decomposed by Discrete Wavelet Transform (DWT). The feature vector of the EEG signals for pattern recognition consists of the coefficients of DWT extracted from the biological signals [30]. Hence these certain frequency bands may provide better classification results for single-channel SSVEP-based biometric recognition [25]. This study used the wavelet type of Sym10 (Symlets) at the 4th level to extract certain frequencies (0-16 $\mathrm{Hz}$ ) over the recorded data with a sampling frequency of $256 \mathrm{~Hz}$.

$$
\operatorname{DWT}(a, b)=\frac{1}{\sqrt{a}} \sum_{n} x(n) \psi^{*}\left(\frac{n-b}{a}\right)
$$

The mathematical relation for DWT is represented in Eq.1, where $a$ and $b$ are named as translation and scaling parameters, respectively [25]. Figure 4 shows the decomposition process for EEG signals.

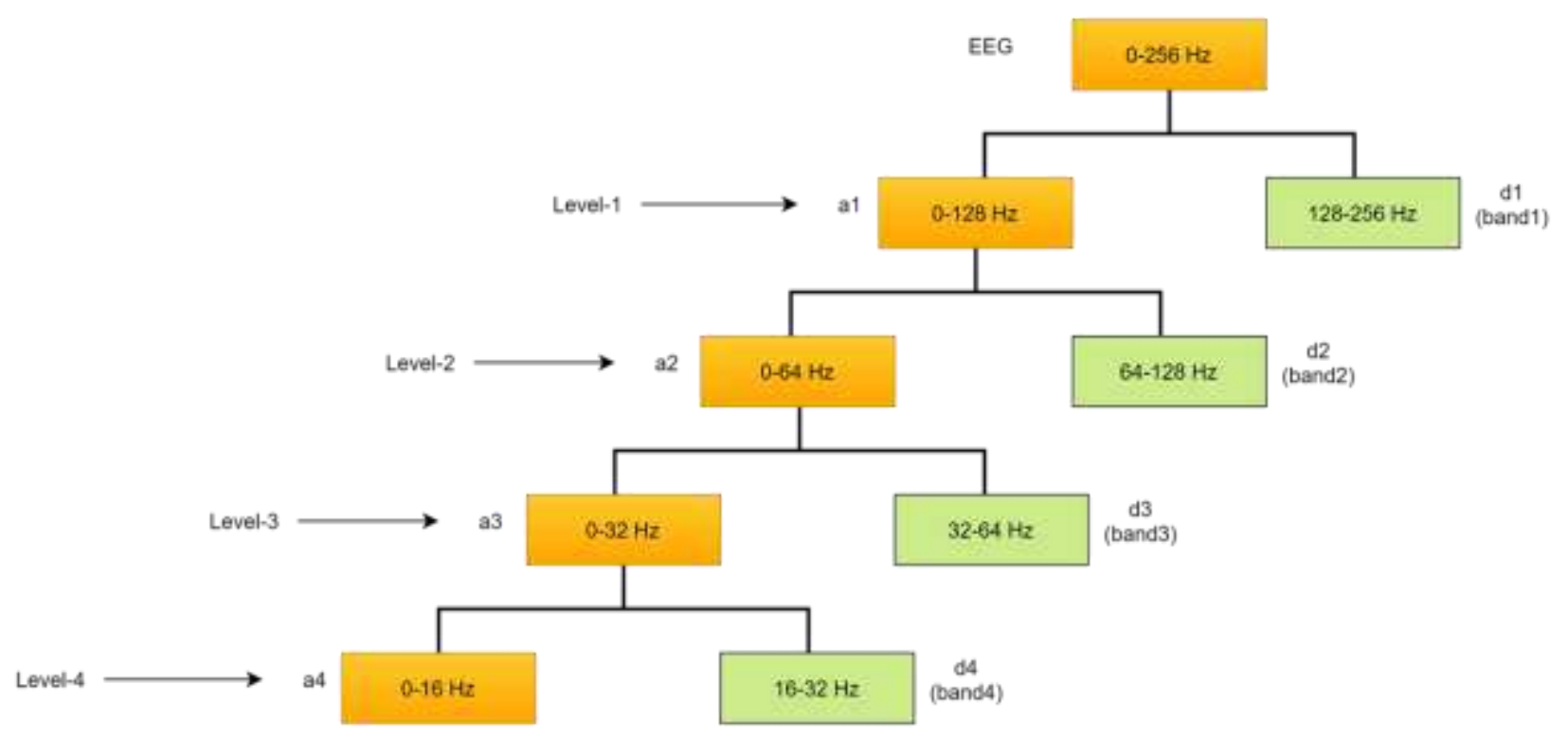

Figure 4 Wavelet decomposition process up to level-4 for the EEG frequency bands

\subsection{Evaluation Metrics}

The evaluation metrics chosen for pattern recognition are crucial to revealing the performances of the deep learning models. The appropriate metrics for this study are listed as; classification accuracy (ACC), sensitivity (SENS), specificity (SPEC), precision (PREC), F-score, 
information transfer rate (ITR), receiver operating characteristics (ROC), and area under the curve $(A \cup C)$.

In this study, traditional validation was implemented to determine the biometric data process as $80 \%$ of the data for training and $20 \%$ for testing. In addition to this validation, the $\mathrm{k}$-fold cross-validation technique has also been examined. The process divides into $k$ subsets to use each separated part for both testing and training. The machine learning model is trained with $k$-1 subsets, and the other remaining subset is carried out for testing [25]. In this work, 3-fold cross-validation was implemented for data processing. Formulas used to calculate classification accuracy are given in Eqs.2-4.

$$
\begin{gathered}
\operatorname{Accuracy}(N)=\frac{\sum_{i=1}^{|N|} \operatorname{estimate}\left(n_{i}\right)}{|N|}, n_{i} \in N \\
\operatorname{Estimate}(n)= \begin{cases}1, & \text { if estimate }(n)=c n \\
0, & \text { otherwise }\end{cases} \\
\text { Classification Accuracy }(M L)=\frac{\sum_{i=1}^{|k|} \operatorname{Accuracy}\left(N_{i}\right)}{|k|}
\end{gathered}
$$

where, $N$ implies the test dataset, $c n$ stands for the class of the value of $n$. Estimate $(n)$ is related to the classification result of $n$, and the $k$ means for the k-fold cross-validation parameter. The accuracy metric alone is commonly seen as an unsatisfactory parameter when determining the proposed model is successful [25]. In this respect, it is useful to explain the statistical terms; specificity (SPEC), sensitivity (SENS), precision (PREC), and F-score. The term "specificity" refers to the ratio of correctly predicted true negatives, while the term "sensitivity" defines the ratio for correctly predicted true positives. The ratio of positive predictions that are correct is named as "precision". F-score is determined via the harmonic mean of the precision and sensitivity [31]. Formulas for SPEC, SENS, PREC, F-score and ITR are given in Eqs.5-9.

$$
\begin{gathered}
S P E C=\frac{T N}{T N+F P} \\
S E N S=\frac{T P}{T P+F N} \\
P R E C=\frac{T P}{T P+F P}
\end{gathered}
$$




$$
\begin{gathered}
F \text {-score }=\frac{2 T P}{2 T P+F P+F N} \\
B=\log _{2} N+P \log _{2} P+(1-P) \log _{2} \frac{(1-P)}{(N-1)}
\end{gathered}
$$

where, these terms are true positive (TP), true negative (TN), the false positive (FP), false negative (FN) $[25,31]$. $B$ means the number of bits per trial, $N$ stands for classes, and the accuracy is named as $P$. The value of the ITR improves, when the number of various classes in a pattern recognition process increases [25]. Moreover, receiver operating characteristic (ROC) and area under the curve (AUC) evaluation metrics are employed to visualize the performance of the deep nets. The ROC curve is plotted as a two-dimensional probability graph with the false positive rate (X-axis) and the true positive rate (Y-axis) [32, 33]. AUC, which refers to the degree of separation between classes, is provided by determining the area under the entire ROC curve. Higher AUC values mean better classifier performances and are calculated in the range of 0 and $1[32,33]$.

\subsection{Long Short-Term Memory Network}

Long Short-Term Memory (LSTM) is a special type of Recurrent Neural Network (RNN) and has an especially ideal structure for sequenced data with long temporal dependencies between them $[34,35]$. This type of network consists of repeating structures in the form of chains.

Let $x_{i} \in \mathbb{R}^{d}$ be given a series of input vector $\left[x_{1}, x_{2}, \ldots, x_{k}\right]$. RNN generates a hidden sequence $\left[h_{1}, h_{2}, \ldots, h_{k}\right]$ at each processing step. The activation of the hidden state at time $t$ is a function of the current input $x_{t}$ and the previous hidden state $h_{t-1}$. This process can be formulated as:

$$
h_{t}=f\left(x_{t}, h_{t-1}\right)
$$

The result of a continuous processing of state information in RNNs is considered unlikely to be transmitted without breaking long-term dependencies. LSTMs are structured to interest longterm dependencies. There are modules consisting of different gate combinations in the LSTM. The names of these gates are given as forgotten gate, input gate, and output gates. The volume of information is determined how much of it will be transmitted to the next stage in the forget gates. The sigmoid function is implemented for this operation. If the result of this function leads to 0 , this means that no information will be transmitted. If it is 1 , the whole information will be transmitted. The next step is carried out for deciding on the information to be stored. This task is accomplished with the sigmoid function at the input gate. It then 
constructs the vector of candidate values $\widetilde{C_{t}}$ implementing the $\tanh$ function. Hence two processes are then combined. After that, the new state information $\left(C_{t}\right)$ for the memory cell is computed. Finally, the system output is calculated [35]. Figure 5 shows the internal structure of the LSTM module. The mathematical notations are given as:

$$
\begin{aligned}
& i_{t}=\sigma\left(W_{i} \cdot\left[h_{t-1}, x_{t}\right]+b_{i}\right) \\
& \tilde{C}_{t}=\tanh \left(W_{C} \cdot\left[h_{t-1}, x_{t}\right]+b_{C}\right) \\
& C_{t}=f_{t} * C_{t-1}+i_{t} * \tilde{C}_{t} \\
& o_{t}=\sigma\left(W_{o} \cdot\left[h_{t-1}, x_{t}\right]+b_{o}\right) \\
& h_{t}=o_{t} * \tanh \left(C_{t}\right)
\end{aligned}
$$

In general, $W$ describes the weight vector, $b$ means the bias term, $\sigma$ is the sigmoid activation function for non-linearity, $x_{t}$ stands for the input sequence, $h_{t-1}$ defines the output of the neuron at time $t-1$ for feedback into the neuron. Moreover, $i_{t}, f_{t}$ and $o_{t}$ are input, forget and output gate, respectively [35]. The new status information of the memory cell must be calculated.

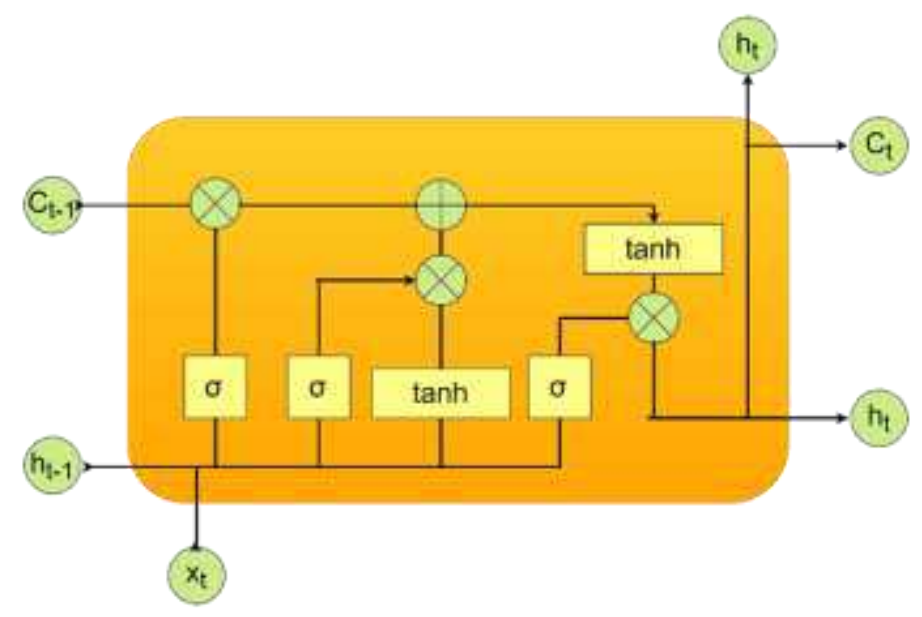

Figure 5 LSTM module internal structure [36]

\subsection{Bidirectional Models and Gated Recurrent Unit}

Representations of previous time steps are learned by LSTM models. However, understanding the content better and eliminating ambiguities are important to learn future representations in different application tasks. Bidirectional models offer a forward propagation process in two stages as main difference according to standard models. Firstly, it starts from the first step, 
and the values up to the last step are computed. Then, starting the process from the last time step to the first time step is performed. Therefore this LSTM model is named as BidirectionalLSTM or (BiLSTM) [37], as shown in Fig.6.

Gated Recurrent Unit (GRU) is designed as a simplified version of the LSTM, and it can be seen that GRU is comparable to LSTM because of the simpler structure and a lower computational complexity. GRU simplifies the structure via merging the cell state and hidden state. This merging process is developed by integrating the forget gate and input gate into a single update gate. When the LSTM structure has three gates (namely forget gate, input gate, and output gate), the GRU only contains two gates (namely reset gate and update gate). Furthermore, the number of parameters of GRU is fewer compared to the LSTM. This leads to less training time and better training loss with fewer training iterations [38].

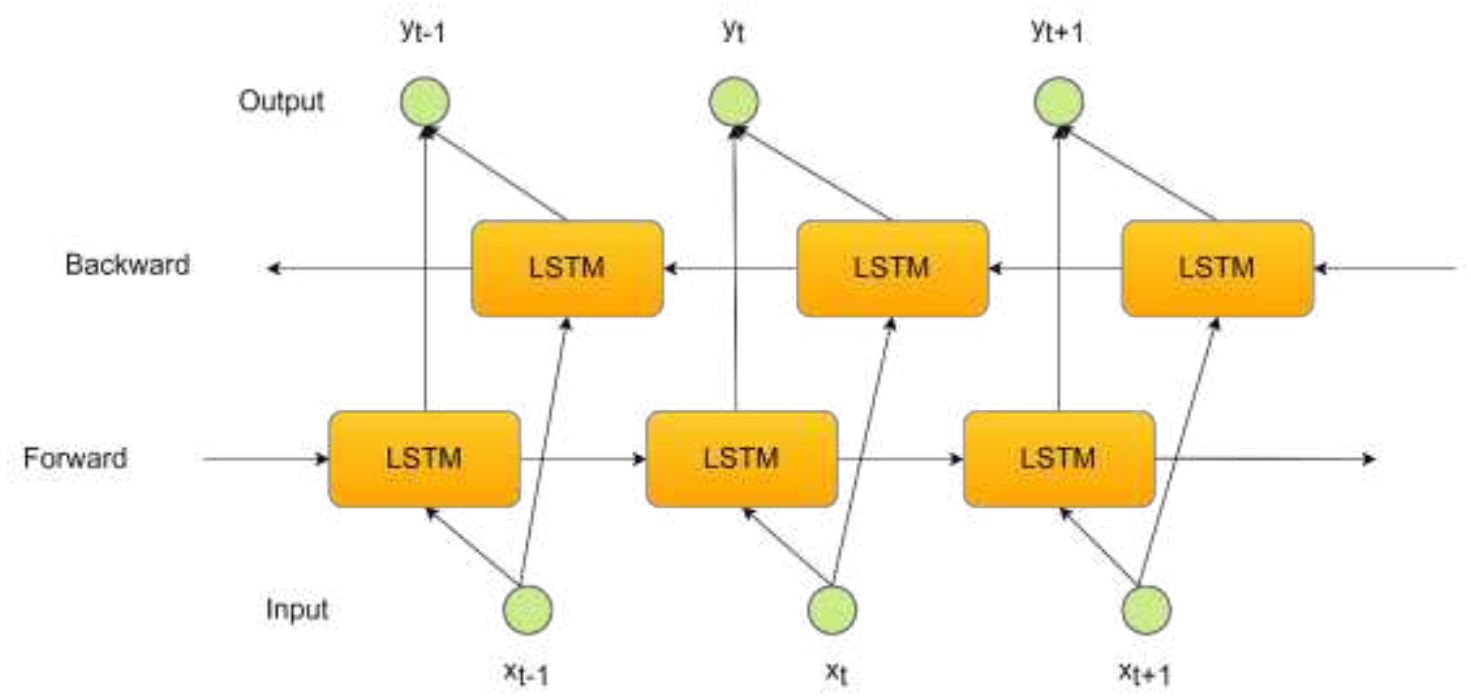

Figure 6 The schematic of the BiLSTM structure

Table 2 Hidden neuron numbers for RNN-based deep models

\begin{tabular}{ccc}
\hline Model-1 & Model-2 & Model-3 \\
\hline 100 & 150 & 200 \\
\hline
\end{tabular}

\section{Results}

Most of the EEG-based biometric works often force the users to perform some activities, including viewing a series of pictures or making/imaging a movement to trigger the signals of 
interest [3]. However, EEG-based biometric systems have some usability issues for real-life scenarios when triggering the signals or multi-channel during acquisition. The actual performance of a biometric system depends on several factors. These are the specific biometric trait, sensor characteristics while capturing, the number and characteristics of the individuals. Moreover, environmental indoor and outdoor factors such as temperature and humidity are also important [1]. However, in this study, the single-channel dry-sensor SSVEPbased biometric approach via RNN-based deep learning investigation can provide a high signal-to-noise ratio and low susceptibility against artifacts as well as real-time and accurate usage without training $[3,10]$. The great results of the research offer a good candidate for biometric recognition among the 11 people with SSVEP responses of the 4 stimulus frequencies.
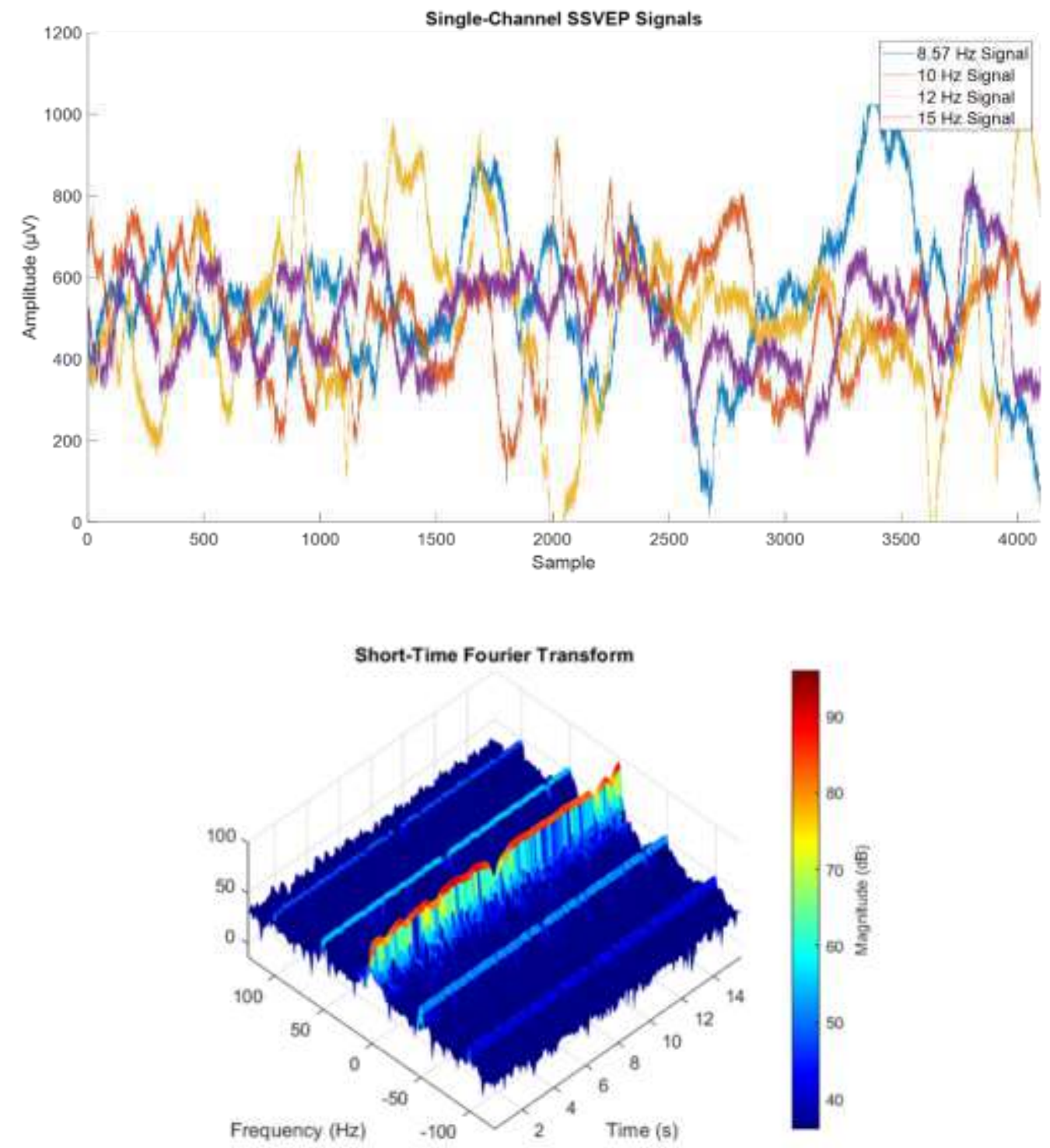
Figure 7 The single-channel raw SSVEP signals (upper), time-frequency analysis for $10 \mathrm{~Hz}$ (lower) (The related evoked potential responses from the Subject-3)

RNN-based deep architectures are specialized in the time series temporal dependencies. However, the size of the signals for EEG is also important to be better recognized by LSTM, BiLSTM, and GRU. Hence, the two sizes of the SSVEP signals are reconstructed, as shown in Figure 3 . These datasets are obtained in the shape of the $64 \times 256$ and $128 \times 128$ matrices for each subject.

The SSVEP signals for 4 stimulus frequencies are processed by RNN-based deep architectures for classic validation (80-20\% and $70-30 \%$ ) and 3-fold cross-validation. The outcomes for the raw SSVEP signals and DWT-based SSVEP signals are presented in the tables and figures:

Table 3 Classic validation results for the size of $64 \times 256$ datasets (RNN Models- 1 )

\begin{tabular}{|c|c|c|c|c|c|c|c|}
\hline & & \multicolumn{3}{|c|}{$80 \%$ validation } & \multicolumn{3}{|c|}{$70 \%$ validation } \\
\hline \multirow{8}{*}{ 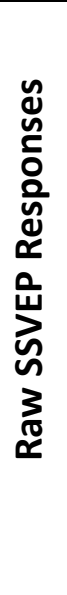 } & $\%$ & BiLSTM & LSTM & GRU & BiLSTM & LSTM & GRU \\
\hline & ACC & 97.90 & 100 & 97.20 & 98.63 & 98.18 & 99.54 \\
\hline & SENS & 100 & 100 & 100 & 100 & 100 & 100 \\
\hline & SPEC & 100 & 100 & 100 & 100 & 100 & 100 \\
\hline & PREC & 97.90 & 100 & 97.20 & 98.63 & 98.18 & 99.54 \\
\hline & F1 & 98.93 & 100 & 98.58 & 99.31 & 99.08 & 99.77 \\
\hline & AUC & 1 & 1 & 1 & 1 & 1 & 1 \\
\hline & ITR & 2.606 & 2.793 & 2.550 & 2.667 & 2.629 & 2.753 \\
\hline \multirow{9}{*}{ 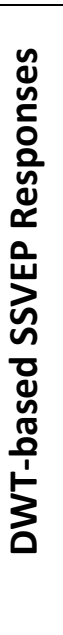 } & & \multicolumn{3}{|c|}{$80 \%$ validation } & \multicolumn{3}{|c|}{$70 \%$ validation } \\
\hline & $\%$ & BiLSTM & LSTM & GRU & BiLSTM & LSTM & GRU \\
\hline & ACC & 99.30 & 100 & 97.20 & 99.54 & 95.00 & 100 \\
\hline & SENS & 100 & 100 & 100 & 100 & 100 & 100 \\
\hline & SPEC & 99.23 & 100 & 100 & 99.50 & 100 & 100 \\
\hline & PREC & 99.30 & 100 & 97.20 & 99.54 & 95.00 & 100 \\
\hline & F1 & 99.64 & 100 & 98.58 & 99.77 & 97.43 & 100 \\
\hline & AUC & 1 & 1 & 1 & 0.961 & 1 & 1 \\
\hline & ITR & 2.729 & 2.793 & 2.550 & 2.753 & 2.391 & 2.793 \\
\hline
\end{tabular}


According to Table 3, LSTM and GRU have provided the highest outcomes at the raw and DWTbased SSVEP signals for all evaluation metrics in $80-20 \%$ and $70-30 \%$ validation, respectively. In addition, it was observed that GRU found a high F-score of $99.77 \%$ as well as $99.54 \%$ ACC, $100 \%$ sense, and spec values for 11 people biometric recognition via single-channel evoked potential. The impact of the sub-band signals via discrete wavelet coefficients has been reported to be slightly higher than the raw signals except for the LSTM model for $70-30 \%$ validation. Figures 8 and 9 show the confusion matrices and ROC curves with t-SEN graph for $80-20 \%$ and $70-30 \%$ traditional validation prediction outcomes for 11 people biometric recognition.
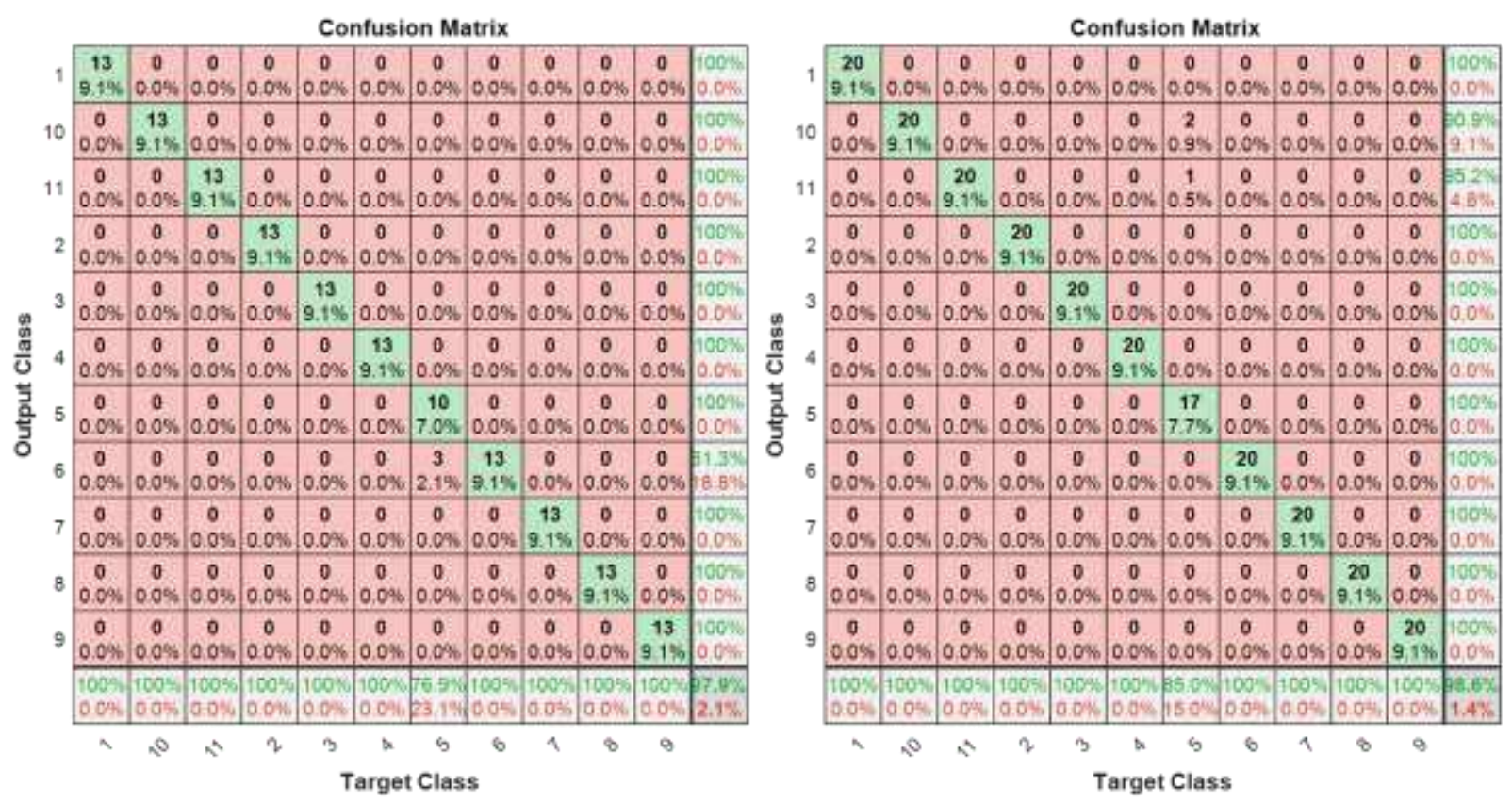

Figure 8 Confusion matrices determined by BiLSTM for $80-20 \%$ (left) and $70-30 \%$ (right) training-testing validation, respectively in raw SSVEP responses 

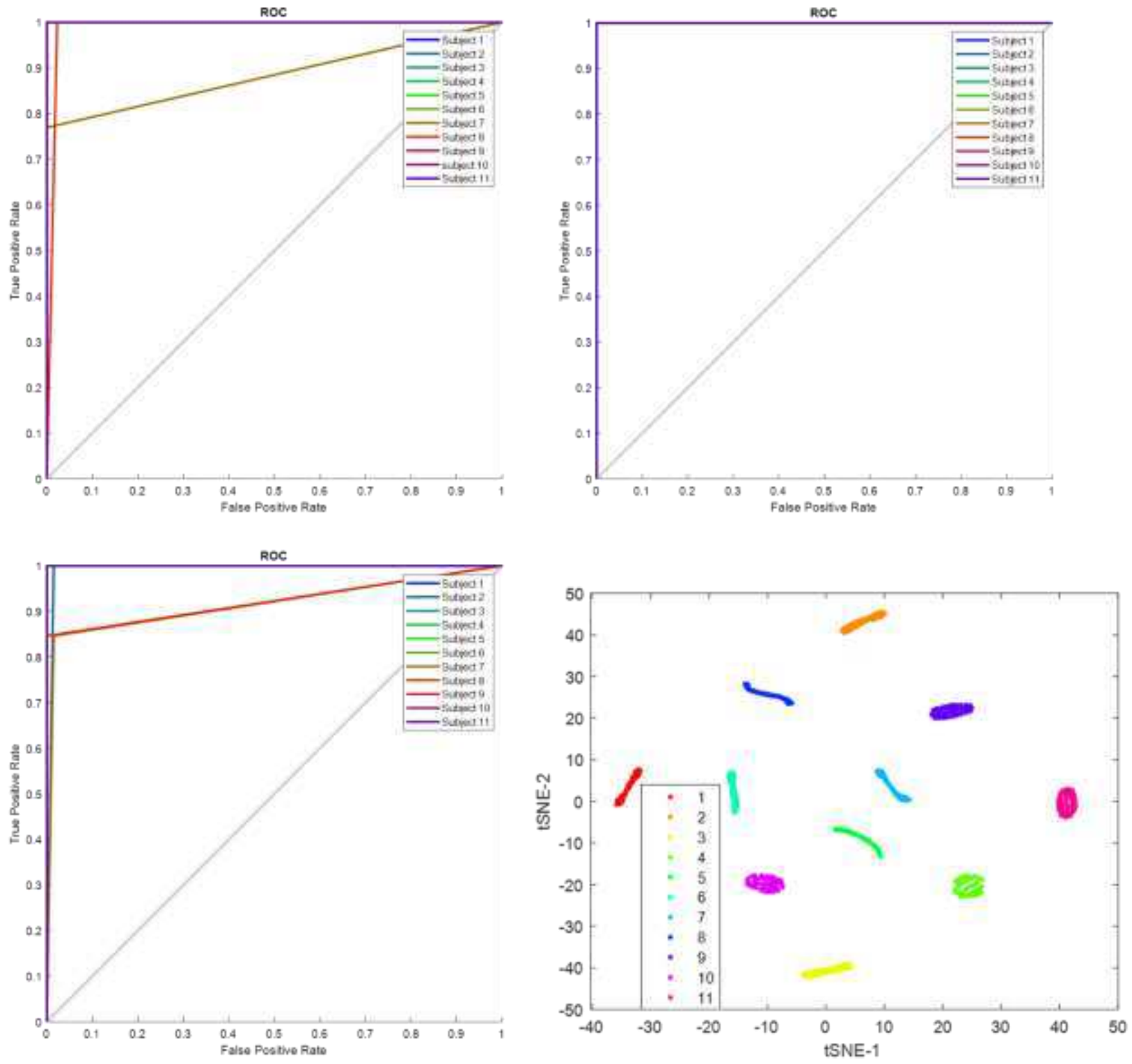

Figure 9 ROC curves and t-distributed stochastic neighbor embedding (t-SNE) presentation for 11 individual biometric recognition 
Table 4 3-fold cross validation results for the size of $128 \times 128$ datasets (RNN Models-2)

\begin{tabular}{|c|c|c|c|c|c|c|c|c|c|}
\hline & \multicolumn{5}{|c|}{ Raw SSVEP Responses } & \multicolumn{4}{|c|}{ DWT-based SSVEP Responses } \\
\hline & $\%$ & fold1 & fold2 & fold3 & Avg. & fold1 & fold2 & fold3 & Avg. \\
\hline \multirow{8}{*}{$\frac{\sum}{n}$} & ACC & 100 & 100 & 100 & 100 & 100 & 100 & 91.00 & 97.00 \\
\hline & SENS & 100 & 100 & 100 & 100 & 100 & 100 & 100 & 100 \\
\hline & SPEC & 100 & 100 & 100 & 100 & 100 & 100 & 100 & 100 \\
\hline & PREC & 100 & 100 & 100 & 100 & 100 & 100 & 91.00 & 97.00 \\
\hline & F1 & 100 & 100 & 100 & 100 & 100 & 100 & 95.29 & 98.43 \\
\hline & AUC & 1 & 1 & 1 & 1 & 1 & 1 & 1 & 1 \\
\hline & ITR & 2.793 & 2.793 & 2.793 & 2.793 & 2.793 & 2.793 & 2.138 & 2.574 \\
\hline & $\%$ & fold1 & fold2 & fold3 & Avg. & fold1 & fold2 & fold3 & Avg. \\
\hline \multirow{8}{*}{$\underset{n}{\Sigma}$} & ACC & 99.14 & 100 & 99.57 & 99.57 & 98.72 & 99.35 & 90.88 & 96.31 \\
\hline & SENS & 100 & 100 & 100 & 100 & 100 & 100 & 100 & 100 \\
\hline & SPEC & 100 & 100 & 100 & 100 & 100 & 100 & 95.33 & 98.44 \\
\hline & PREC & 99.14 & 100 & 99.57 & 99.57 & 98.72 & 99.35 & 90.88 & 96.31 \\
\hline & F1 & 99.57 & 100 & 99.78 & 99.78 & 99.35 & 99.67 & 95.22 & 98.08 \\
\hline & AUC & 1 & 1 & 1 & 1 & 1 & 1 & 1 & 1 \\
\hline & ITR & 2.710 & 2.793 & 2.749 & 2.750 & 2.673 & 2.729 & 2.126 & 2.509 \\
\hline & $\%$ & fold1 & fold2 & fold3 & Avg. & fold1 & fold2 & fold3 & Avg. \\
\hline \multirow{7}{*}{ 롱 } & ACC & 99.57 & 99.14 & 99.14 & 99.28 & 100 & 99.57 & 89.74 & 96.43 \\
\hline & SENS & 100 & 100 & 100 & 100 & 100 & 100 & 100 & 100 \\
\hline & SPEC & 100 & 100 & 100 & 100 & 100 & 100 & 100 & 100 \\
\hline & PREC & 99.57 & 99.14 & 99.14 & 99.28 & 100 & 99.57 & 89.74 & 96.43 \\
\hline & F1 & 99.78 & 99.57 & 99.57 & 99.64 & 100 & 99.78 & 94.59 & 98.12 \\
\hline & AUC & 1 & 1 & 1 & 1 & 1 & 1 & 1 & 1 \\
\hline & ITR & 2.749 & 2.710 & 2.710 & 2.723 & 2.749 & 2.749 & 2.062 & 2.520 \\
\hline
\end{tabular}

At the biometric recognition results of 11 individuals according to Table 4, the BiLSTM model has achieved the highest average and fold performances (100\%) for the metrics of ACC, SENS, SPEC, PREC, and F-score among the other deep architectures in the raw SSVEP signals. The 
same deep architecture has also calculated 97.00 ACC with 1 of AUC score and 2.574 ITR value in the sub-band signals via DWT technique. On the other hand, LSTM and GRU have determined ACC values of over $99 \%$ and an AUC score of 1 , respectively, in the raw SSVEP signals. In general, it is observed that the raw stimulated responses were classified better than the DWT-based SSVEP responses via all RNN-based deep architectures. In a concise comparison, 3-fold cross-validation outcomes were reported to be close to the traditional validation results. Whisker box plot and training process were represented in Figure 10 according to Table 4.
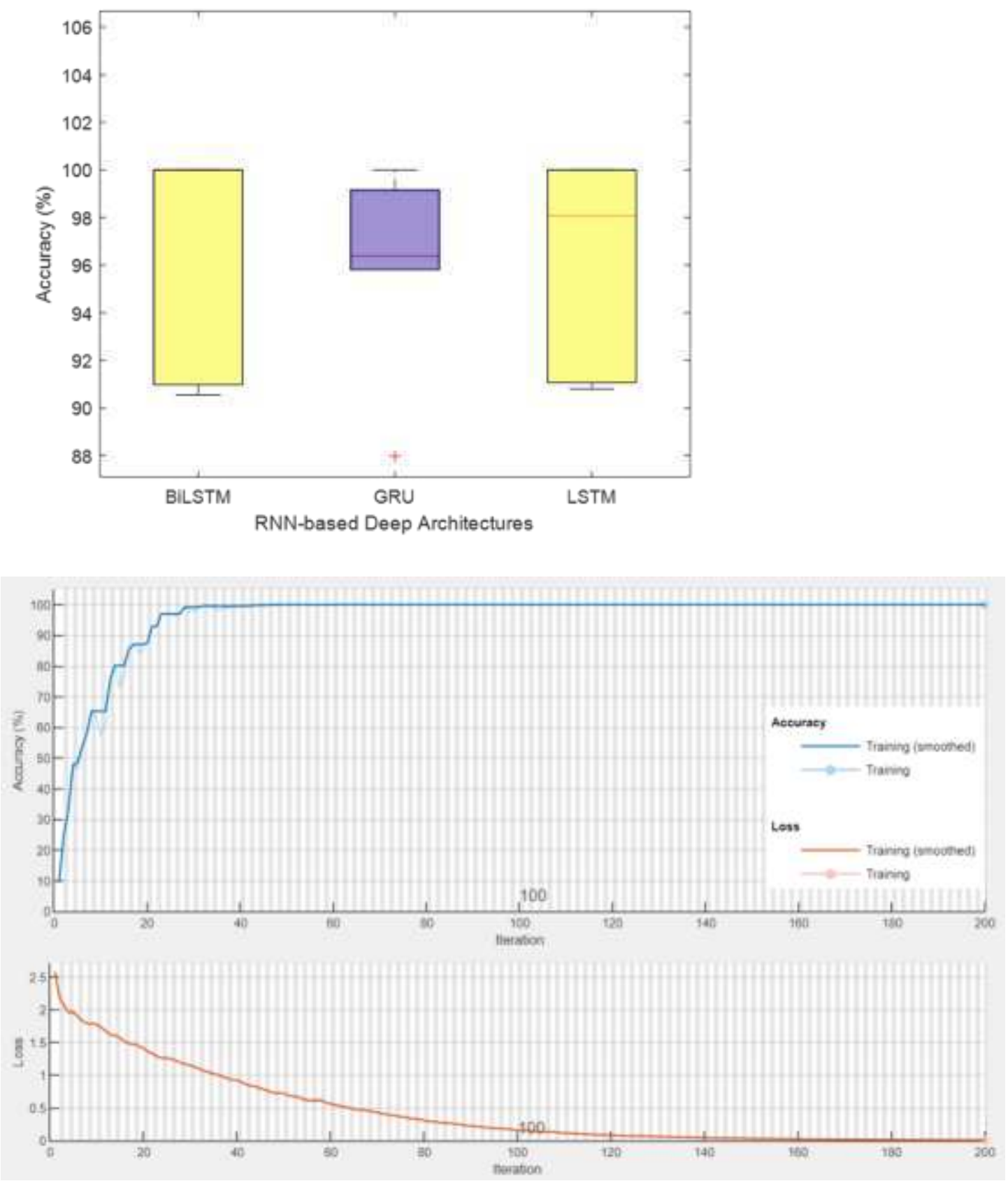
Figure 10 The biometric recognition variations of all RNN-based deep architectures (Whisker box plot) and training process of BiLSTM model in 3-fold cross-validation for the size of $128 \times 128$ dataset

Table 53 -fold cross validation results for the size of $64 \times 256$ datasets (RNN Models-3)

\begin{tabular}{|c|c|c|c|c|c|c|c|c|c|}
\hline & \multicolumn{5}{|c|}{ Raw SSVEP Responses } & \multicolumn{4}{|c|}{ DWT-based SSVEP Responses } \\
\hline & $\%$ & fold1 & fold2 & fold3 & Avg. & fold1 & fold2 & fold3 & Avg. \\
\hline \multirow{8}{*}{$\frac{\sum}{\frac{\Sigma}{n}}$} & ACC & 100 & 100 & 90.55 & 96.85 & 100 & 100 & 90.98 & 96.99 \\
\hline & SENS & 100 & 100 & 100 & 100 & 100 & 100 & 100 & 100 \\
\hline & SPEC & 100 & 100 & 100 & 100 & 100 & 100 & 100 & 100 \\
\hline & PREC & 100 & 100 & 90.55 & 96.85 & 100 & 100 & 90.98 & 96.99 \\
\hline & F1 & 100 & 100 & 95.04 & 98.34 & 100 & 100 & 95.28 & 98.42 \\
\hline & AUC & 1 & 1 & 1 & 1 & 1 & 1 & 1 & 1 \\
\hline & ITR & 2.793 & 2.793 & 2.108 & 2.564 & 2.793 & 2.793 & 2.132 & 2.572 \\
\hline & $\%$ & fold1 & fold2 & fold3 & Avg. & fold1 & fold2 & fold3 & Avg. \\
\hline \multirow{8}{*}{$\underset{n}{\Sigma}$} & ACC & 99.14 & 97.00 & 91.06 & 95.73 & 100 & 100 & 90.79 & 96.93 \\
\hline & SENS & 100 & 100 & 100 & 100 & 100 & 100 & 100 & 100 \\
\hline & SPEC & 100 & 100 & 100 & 100 & 100 & 100 & 100 & 100 \\
\hline & PREC & 99.14 & 97.00 & 91.06 & 95.73 & 100 & 100 & 90.79 & 96.93 \\
\hline & F1 & 99.57 & 98.48 & 95.32 & 97.79 & 100 & 100 & 95.17 & 98.39 \\
\hline & AUC & 1 & 1 & 1 & 1 & 1 & 1 & 1 & 1 \\
\hline & ITR & 2.710 & 2.535 & 2.138 & 2.461 & 2.793 & 2.793 & 2.120 & 2.568 \\
\hline & $\%$ & fold1 & fold2 & fold3 & Avg. & fold1 & fold2 & fold3 & Avg. \\
\hline \multirow{7}{*}{ 兑 } & ACC & 95.81 & 100 & 99.16 & 98.32 & 96.21 & 96.56 & 87.98 & 93.58 \\
\hline & SENS & 100 & 100 & 100 & 100 & 100 & 100 & 100 & 100 \\
\hline & SPEC & 98.15 & 100 & 100 & 99.38 & 100 & 100 & 100 & 100 \\
\hline & PREC & 95.81 & 100 & 99.16 & 98.32 & 96.21 & 96.56 & 87.98 & 93.58 \\
\hline & F1 & 97.86 & 100 & 99.57 & 99.14 & 98.07 & 98.25 & 93.60 & 96.64 \\
\hline & AUC & 1 & 1 & 1 & 1 & 1 & 1 & 1 & 1 \\
\hline & ITR & 2.447 & 2.793 & 2.710 & 2.640 & 2.476 & 2.498 & 1.962 & 2.312 \\
\hline
\end{tabular}


The performance comparison in respect of RNN-based deep architectures for the $64 \times 256$ dataset represents that GRU has carried out the greatest average values reaching the $98.32 \%$ ACC and 1 of AUC score in the raw SSVEP signals. Moreover, the ITR value has been calculated by GRU as 2.640. BiLSTM and LSTM have achieved the second and third best performances in biometric recognition for 11 subjects ' evoked potential signals. However, the highest average performances were observed by BiLSTM (96.99\% ACC and 98.42\% F-score) and LSTM (96.93\% and $98.39 \%$ F-score) deep architectures in the DWT-based SSVEP signals. Furthermore, it is investigated that RNN-based deep architectures have shown better classification performances for 11 subjects in the size of the $128 \times 128$ dataset than the dataset of $64 \times 256$ size. The spider plot presentations are provided for the raw and DWT-based SSVEP signals according to Table 5 .
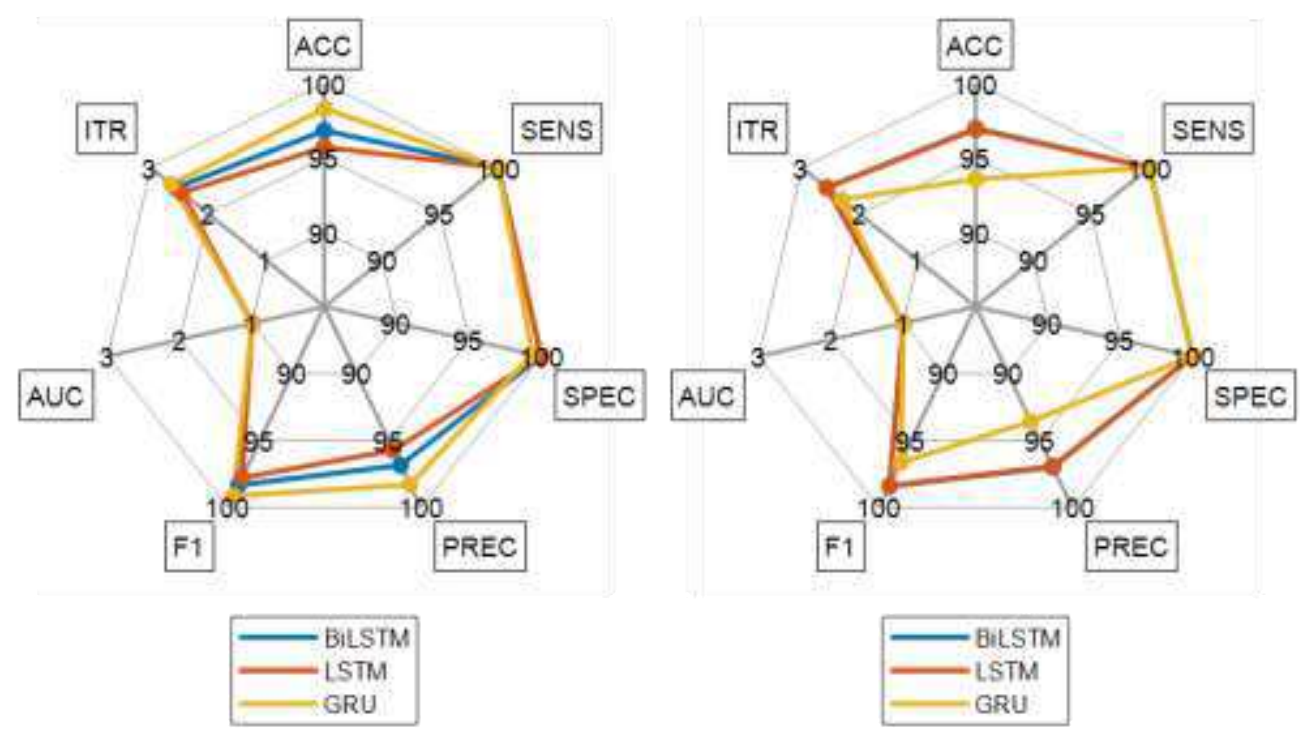

Figure 11 The comparison of the biometric recognition from the raw SSVEP signals and DWTbased SSVEP signals for the size of $64 \times 256$ dataset 
Table 6 Biometric recognition results in 6 indiviuals for the size of $128 \times 128$ dataset (RNN Models-2)

\begin{tabular}{|c|c|c|c|c|}
\hline $80 \%$ validation & $\%$ & BiLSTM & LSTM & GRU \\
\hline \multirow{7}{*}{ 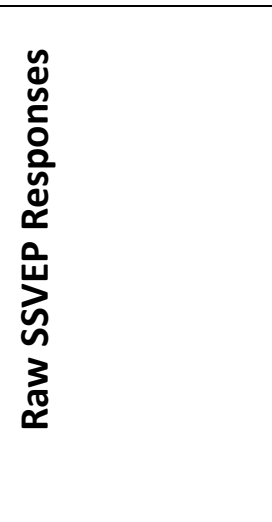 } & ACC & 100 & 98.07 & 94.87 \\
\hline & SENS & 100 & 100 & 100 \\
\hline & SPEC & 100 & 100 & 100 \\
\hline & PREC & 100 & 98.07 & 94.87 \\
\hline & F1 & 100 & 99.02 & 97.36 \\
\hline & AUC & 1 & 1 & 1 \\
\hline & ITR & 2.793 & 2.614 & 2.378 \\
\hline
\end{tabular}

With respect to the 6 out of 11 individual biometric recognition, BiLSTM has the highest outcomes for all metrics. Then LSTM and GRU have achieved the second $198.07 \%$ ACC and 99.02\% F-score) and third (94.87\% ACC and 97.36\% F-score) prediction performances. Sensitivity and specificity values are also determined as $100 \%$ for all RNN-based deep architectures. 6 individual biometric classifications are shown in Figure 12.

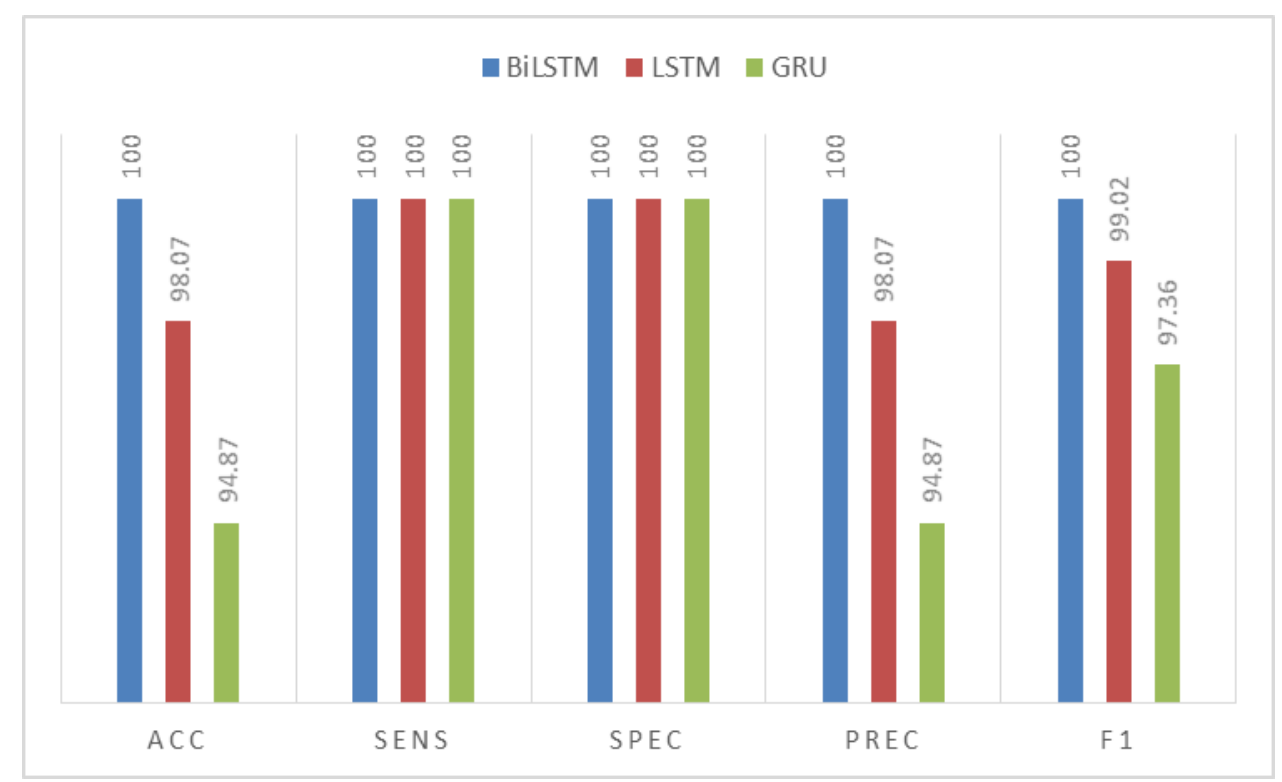




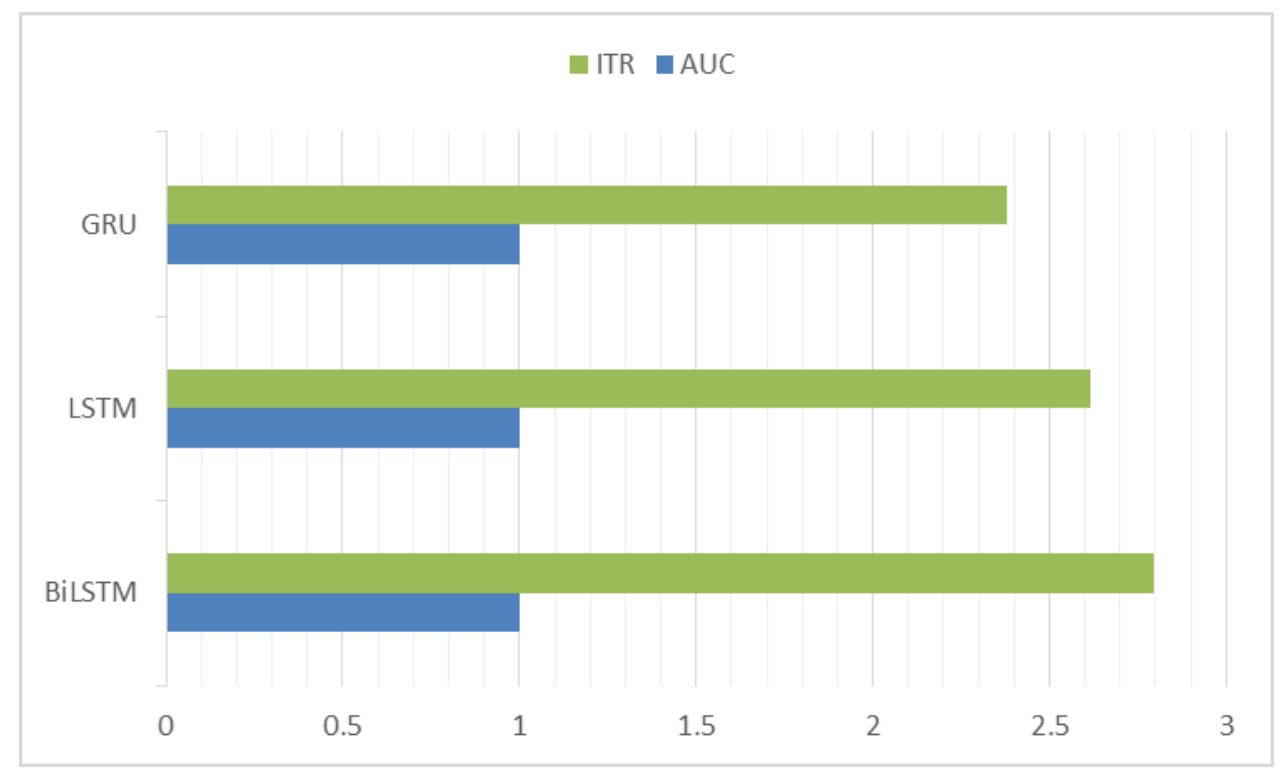

Figure 12 The graphical representation of the single-channel SSVEP-based biometric results for 6 individuals

\section{Discussion}

It is noticeable that EEG-based biometric systems with multi-channels pose the most relevant unsolved problems in real-world usage [39]. According to the biometric recognition results of 11 individuals, the single-channel and single-trial SSVEP signals stimulated via four distinct flickering frequencies provided a proof-of-concept for a robust and easy-to-use biometric system implementing the RNN-based deep architectures.

Our research also focused on the biometric performance and investigation of the RNN-based deep architectures and sub-band effect for different-sized datasets of the single-channel SSVEP responses. First, the biometric recognition processes were conducted via the traditional $80-20 \%$ and $70-30 \%$ validation (Table 3 ). The accuracy rates and the other evaluation metrics of the proposed deep architectures were remarkably in the range of 95-100\% (ACC, SENS, SPEC, PREC, and F-score). The AUC scores and ITR values acquired were in the range of 0.9611 and 2.391-2.793, respectively. It is obvious that the results determined for the $80-20 \%$ and 70-30\% validation and the raw and DWT-based features of the SSVEP responses were close. However, a few successful variations of the deep architectures (BiLSTM, LSTM, and GRU) were also observed for both datasets. The 3-fold results and the average of those for the differentsized datasets (Tables 4 and 5) also offered great biometric recognition over 11 individualevoked potentials. The average accuracy range (96.31-100\%) and the other evaluation metrics demonstrated that the datasets of $128 \times 128$ in size, consisting of raw and DWT-based 
features, provided better biometric recognition outcomes for 11 persons compared to the datasets of $64 \times 256$ in size (93.58-98.32\% average accuracy). Furthermore, when the number of participants (Table 6) was reduced to six individuals, the biometric recognition results did not significantly increase or change (94.87-100\% ACC and $97.36-100 \%$ F-score). This may prove that the RNN-based deep architectures had determined robust biometric recognition over the single-channel SSVEP responses for both numbers of individuals. The ROC curves, t-SEN, and confusion matrices presented the same achievements.

The flickering frequency set of $8.57,10,12$, and $15 \mathrm{~Hz}$ in the single-channel SSVEP-based biometric approach can cause frequency dependence of the SSVEP amplitude. Moreover, significant variabilities in SSVEP responses for individuals in terms of the amplitude and peak values have been reported [40]. In this study, a resizing and merging process for the flickering frequencies was implemented to overcome these deficiencies. Hence, the success of the single-channel SSVEP-based biometric approach for 11 people may have been dependent on this implemented task. By using 12 and $15 \mathrm{~Hz}$ in our approach, the suggested frequency range $(12-18 \mathrm{~Hz})$ to provide maximum accuracy for $\mathrm{BCl}$ applications was included [40]. Furthermore, multi-channel recordings were also addressed and found to be helpful in capturing the high flickering frequencies as SSVEP responses [40]. However, the success of the flickering and low frequencies were not investigated for a single-channel SSVEP-based biometric approach using RNN-based deep architectures. Although Oz-Pz placement is generally recommended in the current literature as it produces better and stronger SSVEP responses, it has been noted that the optimum electrode placement can vary for individuals [40]. In the experiment, the EEG signals on the Oz-Fpz electrode pairs were recorded to detect the single-channel SSVEP responses. The electrode placement may have led to the high accuracy rates (up to 100\%) and recognition results obtained in the single-channel SSVEP biometric approach.

The properties of the EEG signals are noted as being non-stationary and non-linear in form. This can make biometric pattern recognition difficult in both the time and frequency domains. Hence, the DWT decomposes these non-stationary signals at different intervals of frequencies with various resolutions to be captured in a more useful part [41]. In our study, 4-level DWT decomposition (Sym10) did not provide better classification success for all sizes of the datasets using RNN-based deep architectures. This may have resulted from the characteristics of the single-channel SSVEP signals. This kind of EEG signal has been reported to exhibit a robust 
characteristic against noise and to provide high signal-to-noise ratio (SNR) values with clearly detectable flickering frequencies [10]. This may have led to the close biometric recognition success of the datasets for raw signal responses $(8.57-15 \mathrm{~Hz})$ and DWT-based signals $(0-16 \mathrm{~Hz})$.

An additional analysis was carried out to verify the effect of the different hidden neuron numbers used by the RNN-based deep architectures (BiLSTM, LSTM, and GRU) on the raw and DWT-based SSVEP signals. According to the proposed neuron numbers (100, 150, and 200), the BiLSTM and GRU achieved better biometric recognition over the DWT-based features for the traditional validation (see Table 3) via RNN model-1. On the other hand, RNN model-2 revealed better average and fold classification results over the raw SSVEP responses. Furthermore, except for the GRU over the DWT-based sub-band signals, RNN model-3 had few successful increments for the biometric recognition of the 11 individuals. These outcomes may reveal that the increment of the feature vector (from 128 to 256 in size) had been applied to the RNN-based deep architectures. The higher neuron numbers can help the models to learn long-term dependencies via time-series signals over the sub-band signals [42]. Hence, the capabilities and effectiveness of the deep neural networks can be improved by the neuron numbers and the different sizes of the datasets in order to identify better biometric patterns over the raw and DWT-based SSVEP signals.

Table 7 Overview of the state-of-the-art EEG-based biometric studies in recent years

\begin{tabular}{|c|c|c|c|c|c|c|c|}
\hline Study/Year & $\begin{array}{c}\text { EEG and } \\
\text { Dataset } \\
\text { Type }\end{array}$ & NFF & SB & $\mathrm{CN}$ & $\begin{array}{l}\text { Pre. and FE } \\
\text { Methods }\end{array}$ & MLs & Perf. \\
\hline$[22] / 2017$ & SSVEP & 4 & 25 & 19 & $\begin{array}{l}\text { Mel-Frequency } \\
\text { Cepstral } \\
\text { Coefficients } \\
\text { (MFCCs) and } \\
\text { Autoregressive } \\
\text { (AR) Reflection } \\
\text { Coefficients }\end{array}$ & $\begin{array}{l}\text { Manhattan } \\
\text { Distance }\end{array}$ & $\begin{array}{c}\text { ACC: } 91.47- \\
96 \%\end{array}$ \\
\hline$[43] / 2018$ & SSVEP & 3 & $\begin{array}{l}5- \\
12\end{array}$ & 8 & $\begin{array}{l}\text { Filtering } \\
\text { Process }\end{array}$ & $\begin{array}{l}\text { CNN, SVM, } \\
\text { Random } \\
\text { Forest (RF) } \\
\text { and NN }\end{array}$ & $\begin{array}{c}\text { ACC: } 80.48- \\
98.10 \%\end{array}$ \\
\hline$[11] / 2019$ & $\begin{array}{l}\text { SSVEP and } \\
\text { ERPs }\end{array}$ & 1 & 20 & 6 & $\begin{array}{c}\text { Filtering } \\
\text { Process and } \\
\text { Cross- } \\
\text { Correlation } \\
\text { Analysis (CCA) }\end{array}$ & LSTM & $\begin{array}{c}\text { ACC: } \\
91.44 \%\end{array}$ \\
\hline
\end{tabular}




\begin{tabular}{|c|c|c|c|c|c|c|c|}
\hline$[4] / 2019$ & SSVEP & 40 & 8 & 9 & $\begin{array}{l}\text { Filtering } \\
\text { Process }\end{array}$ & CNN & ACC: $>97 \%$ \\
\hline$[44] / 2019$ & $\begin{array}{l}\text { c-VEP } \\
\text { (Code } \\
\text { Modulate } \\
\text { d VEPs) }\end{array}$ & 1 & 25 & 9 & $\begin{array}{c}\text { Filter Banks } \\
\text { and CCA }\end{array}$ & $\begin{array}{c}\text { Task- } \\
\text { Related } \\
\text { Component } \\
\text { Analysis }\end{array}$ & $\begin{array}{c}\text { ACC: } 98.43- \\
100 \%\end{array}$ \\
\hline [39]/2020 & $\begin{array}{l}\text { Motor } \\
\text { Imagery } \\
\text { EEGs }\end{array}$ & - & $\begin{array}{c}10 \\
9\end{array}$ & 64 & $\begin{array}{c}\text { Empirical Mode } \\
\text { Decomposition } \\
\text { (EMD) and } \\
\text { DWT }\end{array}$ & $\begin{array}{l}\text { Support } \\
\text { Vector } \\
\text { Machine } \\
\text { (SVM) }\end{array}$ & $\begin{array}{c}\text { TAR: } 0.822- \\
0.927 \\
\text { TRR: } 0.981- \\
0.989\end{array}$ \\
\hline$[45] / 2020$ & $\begin{array}{l}\text { EEG } \\
\text { Signals } \\
\text { Elicited by } \\
\text { Music and } \\
\text { Video }\end{array}$ & - & 32 & 32 & $\begin{array}{c}\text { Filtering } \\
\text { Process and } \\
\text { Mesh Feature } \\
\text { Technique }\end{array}$ & $\begin{array}{l}\text { CNN-LSTM } \\
\text { and CNN- } \\
\text { GRU and } \\
\text { SVM }\end{array}$ & $\begin{array}{c}\text { ACC: } 99.90- \\
100 \%\end{array}$ \\
\hline$[46] / 2020$ & $\begin{array}{c}\text { Mental } \\
\text { Task EEGs }\end{array}$ & - & 45 & 19 & $\begin{array}{c}\text { Common } \\
\text { Average } \\
\text { Referencing } \\
\text { Filtering }\end{array}$ & $\begin{array}{l}\text { CNN, RNN, } \\
\text { LDA and } \\
\text { SVM }\end{array}$ & ACC: $>96 \%$ \\
\hline$[47] / 2021$ & VEP & - & 70 & 14 & $\begin{array}{c}\text { Filtering } \\
\text { Process for } \\
\text { Extracting } \\
\text { Alpha Bands }\end{array}$ & $\begin{array}{l}\text { CNN and } \\
\text { LSTM }\end{array}$ & $\begin{array}{c}\text { ACC: } \\
81.78 \% \\
\text { FAR: } \\
\text { 29.28\% } \\
\text { FRR: } 7.14 \%\end{array}$ \\
\hline$[17] / 2021$ & $\begin{array}{c}\text { Mental } \\
\text { Task EEGs }\end{array}$ & - & 45 & 19 & $\begin{array}{l}\text { Filtering } \\
\text { Process }\end{array}$ & CNN & $\begin{array}{c}\text { EER: } 10.5- \\
16.4 \%\end{array}$ \\
\hline$[48] / 2021$ & $\begin{array}{l}\text { SSVEP and } \\
\text { P300 }\end{array}$ & 4 & 10 & $\begin{array}{l}3- \\
12\end{array}$ & $\begin{array}{l}\text { Filtering } \\
\text { Process and } \\
\text { Canonical } \\
\text { Correlation } \\
\text { (CCA) }\end{array}$ & $\begin{array}{l}\text { Bayesian } \\
\text { Linear } \\
\text { Discriminan } \\
\text { t analysis } \\
\text { (BLDA) }\end{array}$ & $\begin{array}{c}\text { ACC: } 94.61- \\
97.63 \%\end{array}$ \\
\hline$[20] / 2021$ & $\begin{array}{l}\text { VEPs } \\
\text { (Code } \\
\text { Modulate } \\
\text { d VEPs) }\end{array}$ & 2 & 15 & 9 & $\begin{array}{l}\text { Filtering } \\
\text { Process }\end{array}$ & $\begin{array}{l}\text { State-of- } \\
\text { the-Art } \\
\text { Task- } \\
\text { Related } \\
\text { Component } \\
\text { Analysis } \\
\text { (TRCA) }\end{array}$ & $\begin{array}{c}\text { ACC: } 98.93- \\
100 \%\end{array}$ \\
\hline [49]/2021 & $\begin{array}{l}\text { SSVEP, } \\
\text { ERP, MI } \\
\text { and } \\
\text { Hybrid } \\
\text { Signals }\end{array}$ & 4 & 54 & 32 & $\begin{array}{l}\text { Filter Bank } \\
\text { Common } \\
\text { Spatial Pattern } \\
\text { (FBCSP) } \\
\text { Algorithm }\end{array}$ & SVM, CNN & $\begin{array}{c}\text { FRR: } 0.12- \\
7.36 \\
\text { FAR: } 0.27- \\
4.55\end{array}$ \\
\hline$[50] / 2021$ & SSVEP & 2 & 15 & 30 & $\begin{array}{l}\text { Filtering } \\
\text { Process }\end{array}$ & SVM & $\begin{array}{c}\text { TAR: } 84.12- \\
95.51 \% \\
\text { FAR: } 0.7-2.7\end{array}$ \\
\hline
\end{tabular}




\begin{tabular}{|c|c|c|c|c|c|c|c|}
\hline & & & & & $\begin{array}{c}\text { and } \\
\text { Independent } \\
\text { Principal } \\
\text { Component } \\
\text { Analysis }\end{array}$ & & \\
\hline Our Study & SSVEP & 4 & 11 & 1 & $\begin{array}{c}\text { Discrete } \\
\text { Wavelet } \\
\text { Transform and } \\
\text { Data Resizing } \\
\text { Process }\end{array}$ & $\begin{array}{c}\text { LSTM, } \\
\text { BiLSTM and } \\
\text { GRU }\end{array}$ & $\begin{array}{c}\text { ACC: } 93.58 \\
100 \% \\
\text { F1: } 96.64- \\
100 \% \\
\text { ITR: } 2.312- \\
2.793\end{array}$ \\
\hline
\end{tabular}

SB: Subject Number, NFF: Number of Flickering Frequencies CN: Channel Number, Pre: Pre-processing/ FE: Feature Extraction, ML: Machine Learning Models, Perf: Performances, TAR: True Acceptance Rate, TRR: True Rejection Rate, EER: Equal Error Rate

According to the literature survey (Table 7), the single-channel SSVEP-based biometric approach offers highly promising biometric recognition results compared to the multi-channel and multi-trial EEG-based biometrics. It is obvious that the accuracy range in our singlechannel (93.58-100\%) SSVEP-based biometric study is a significant achievement in view of the results of the other studies. It is worth noting that, with the single-channel performance of the $\mathrm{Oz}$ electrode with RNN-based deep architectures, our approach was comparable and provided better robust outcomes for raw signals and DWT-based features. It is crucial for SSVEP-based $\mathrm{BCl}$ studies that the test subjects are untrained and that small data block lengths are used [51]. Only one session and one trial were implemented and $16 \mathrm{~s}$ were allotted for each flickering frequency in the training models. Hence, our approach can offer these important features with single-channel SSVEP-based biometrics using RNN-based deep models.

\section{Conclusion}

Several light sources flickering at different frequencies and their responses on the EEG signals, i.e., SSVEP signals, can provide a highly promising and robust biometric approach. In this study, a single-channel and single-trial SSVEP-based biometric approach was developed using RNNbased deep architectures. Moreover, the biometric recognition results for 11 individuals were investigated and compared systematically for different-sized time series of raw signals and sub-bands signals. To date, the literature had lacked research using a single-channel SSVEPbased biometric approach with a systematic comparison using RNN-based deep architectures. 
Hence, the single-channel, untrained, and singular dynamic of the SSVEP signals for each person can provide a more economical (due to the low set-up cost), easy-to-use, reliable, and high-accuracy (approaching 100\%) biometric approach for real-time usage instead of the traditional static biometrics (face, fingerprints, etc.). In addition, this technique can serve as an important alternative to the noise-prone multi-channel EEG-based biometric methods.

\section{Funding}

This work did not receive any grant from funding agencies in the public, commercial, or notfor-profit sectors.

\section{CRedit authorship contribution statement}

K. Gorur: Conceptualization, Methodology, Software, Validation, Investigation, Writing original draft. B. Eraslan: Writing - review \& editing.

\section{Declaration of competing interest}

The authors declare that they have no conflict of interest.

\section{References}

1. Jain AK (2007) Biometric recognition. Nature 449:38-40. https://doi.org/10.1038/449038a

2. Dargan S, Kumar M (2020) A comprehensive survey on the biometric recognition systems based on physiological and behavioral modalities. Expert Syst Appl 143:113114. https://doi.org/10.1016/j.eswa.2019.113114

3. Yang S, Hoque S, Deravi F (2019) Improved Time-Frequency Features and Electrode Placement for EEG-Based Biometric Person Recognition. IEEE Access 7:49604-49613. https://doi.org/10.1109/ACCESS.2019.2910752

4. Yu T, Wei C-S, Chiang K-J, et al (2019) EEG-Based User Authentication Using a Convolutional Neural Network. In: 2019 9th International IEEE/EMBS Conference on Neural Engineering (NER). IEEE, pp 1011-1014

5. Jayarathne I, Cohen M, Amarakeerthi S (2020) Person identification from EEG using various machine learning techniques with inter-hemispheric amplitude ratio. PLoS One 
15:e0238872. https://doi.org/10.1371/journal.pone.0238872

6. Gorur K, Bozkurt MR, Bascil MS, Temurtas F (2018) Glossokinetic potential based tongue-machine interface for 1-D extraction. Australas Phys Eng Sci Med 41:379-391. https://doi.org/10.1007/s13246-018-0635-x

7. Casson AJ, Smith S, Duncan JS, Rodriguez-Villegas E (2008) Wearable EEG: what is it, why is it needed and what does it entail? In: 2008 30th Annual International Conference of the IEEE Engineering in Medicine and Biology Society. IEEE, pp 5867-5870

8. Acampora G, Trinchese P, Vitiello A (2021) A dataset of EEG signals from a singlechannel SSVEP-based brain computer interface. Data $\mathrm{Br}$ 35:106826. https://doi.org/10.1016/j.dib.2021.106826

9. Singhal GK, RamKumar P (2007) Person Identification Using Evoked Potentials and Peak Matching. In: 2007 Biometrics Symposium. IEEE, pp 1-6

10. Angrisani L, Arpaia P, Casinelli D, Moccaldi N (2019) A Single-Channel SSVEP-Based Instrument With Off-the-Shelf Components for Trainingless Brain-Computer Interfaces. IEEE Trans Instrum Meas 68:3616-3625. https://doi.org/10.1109/TIM.2018.2882115

11. Puengdang S, Tuarob S, Sattabongkot T, Sakboonyarat B (2019) EEG-Based Person Authentication Method Using Deep Learning with Visual Stimulation. In: 2019 11th International Conference on Knowledge and Smart Technology (KST). IEEE, pp 6-10

12. Autthasan P, Du X, Arnin J, et al (2020) A Single-Channel Consumer-Grade EEG Device for Brain-Computer Interface: Enhancing Detection of SSVEP and Its Amplitude Modulation. IEEE Sens J 20:3366-3378. https://doi.org/10.1109/JSEN.2019.2958210

13. Carrión-Ojeda D, Fonseca-Delgado R, Pineda I (2021) Analysis of factors that influence the performance of biometric systems based on EEG signals. Expert Syst Appl 165:113967. https://doi.org/10.1016/j.eswa.2020.113967

14. Behera SK, Kumar P, Dogra DP, Roy PP (2021) A Robust Biometric Authentication System for Handheld Electronic Devices by Intelligently Combining 3D Finger Motions and Cerebral Responses. IEEE Trans Consum Electron 67:58-67. https://doi.org/10.1109/TCE.2021.3055419 
15. Kumar MG, Narayanan S, Sur M, Murthy HA (2021) Evidence of Task-Independent Person-Specific Signatures in EEG Using Subspace Techniques. IEEE Trans Inf Forensics Secur 16:2856-2871. https://doi.org/10.1109/TIFS.2021.3067998

16. Palaniappan R (2004) Method of identifying individuals using VEP signals and neural network. IEE Proc - Sci Meas Technol 151:16-20. https://doi.org/10.1049/ipsmt:20040003

17. Maiorana E (2021) Learning deep features for task-independent EEG-based biometric $\begin{array}{llll}\text { verification. } & \text { Pattern } & \text { Recognit }\end{array}$ https://doi.org/10.1016/j.patrec.2021.01.004

18. Palaniappan R, Mandic DP (2007) Biometrics from Brain Electrical Activity: A Machine Learning Approach. IEEE Trans Pattern Anal Mach Intell 29:738-742. https://doi.org/10.1109/TPAMI.2007.1013

19. Palaniappan R, Ravi KVR (2006) Improving visual evoked potential feature classification for person recognition using PCA and normalization. Pattern Recognit Lett 27:726-733. https://doi.org/10.1016/j.patrec.2005.10.020

20. Zhao H, Chen Y, Pei W, et al (2021) Towards online applications of EEG biometrics using visual evoked potentials. Expert Syst Appl 177:114961. https://doi.org/10.1016/j.eswa.2021.114961

21. Min B-K, Suk $\mathrm{H}-\mathrm{I}$, Ahn $\mathrm{M}-\mathrm{H}$, et al (2017) Individual Identification Using Cognitive Electroencephalographic Neurodynamics. IEEE Trans Inf Forensics Secur 12:2159-2167. https://doi.org/10.1109/TIFS.2017.2699944

22. Piciucco E, Maiorana E, Falzon O, et al (2017) Steady-State Visual Evoked Potentials for EEG-Based Biometric Identification. In: 2017 International Conference of the Biometrics Special Interest Group (BIOSIG). IEEE, pp 1-5

23. Thomas J, Maszczyk T, Sinha N, et al (2017) Deep learning-based classification for braincomputer interfaces. In: 2017 IEEE International Conference on Systems, Man, and Cybernetics (SMC). IEEE, pp 234-239

24. LeCun $Y$, Bengio $Y$, Hinton G (2015) Deep learning. Nature 521:436-444. 
https://doi.org/10.1038/nature14539

25. Gorur K, Bozkurt M, Bascil M, Temurtas F (2019) GKP Signal Processing Using Deep CNN and SVM for Tongue-Machine Interface. Trait du Signal 36:319-329. https://doi.org/10.18280/ts.360404

26. Kim B-H, Pyun J-Y (2020) ECG Identification For Personal Authentication Using LSTMBased Deep Recurrent Neural Networks. Sensors 20:3069. https://doi.org/10.3390/s20113069

27. Kumar S, Sharma A, Tsunoda T (2019) Brain wave classification using long short-term memory network based OPTICAL predictor. Sci Rep 9:9153. https://doi.org/10.1038/s41598-019-45605-1

28. Ruffini G, Ibañez D, Castellano M, et al (2019) Deep Learning With EEG Spectrograms in Rapid Eye Movement Behavior Disorder. Front Neurol 10:. https://doi.org/10.3389/fneur.2019.00806

29. Kaushik P, Gupta A, Roy PP, Dogra DP (2019) EEG-Based Age and Gender Prediction Using Deep BLSTM-LSTM Network Model. IEEE Sens J 19:2634-2641. https://doi.org/10.1109/JSEN.2018.2885582

30. Chen D, Wan S, Xiang J, Bao FS (2017) A high-performance seizure detection algorithm based on Discrete Wavelet Transform (DWT) and EEG. PLoS One 12:e0173138. https://doi.org/10.1371/journal.pone.0173138

31. Abdar M, Yen NY, Hung JC-S (2018) Improving the Diagnosis of Liver Disease Using Multilayer Perceptron Neural Network and Boosted Decision Trees. J Med Biol Eng 38:953-965. https://doi.org/10.1007/s40846-017-0360-z

32. Fawcett T (2006) An introduction to ROC analysis. Pattern Recognit Lett 27:861-874. https://doi.org/10.1016/j.patrec.2005.10.010

33. Metz CE (1978) Basic principles of ROC analysis. Semin Nucl Med 8:283-298. https://doi.org/10.1016/S0001-2998(78)80014-2

34. Ozer I, Cetin O, Gorur K, Temurtas F (2021) Improved machine learning performances with transfer learning to predicting need for hospitalization in arboviral infections 
against the small dataset. Neural Comput Appl 33:14975-14989. https://doi.org/10.1007/s00521-021-06133-0

35. Ozer I, Efe SB, Ozbay H (2021) A combined deep learning application for short term load forecasting. Alexandria Eng J 60:3807-3818. https://doi.org/10.1016/j.aej.2021.02.050

36. BAYRAM MA, ÖZER I, TEMURTAŞ F (2021) Deep Learning Methods for Autism Spectrum Disorder Diagnosis Based on fMRI Images. Sak Univ J Comput Inf Sci 4:142-155. https://doi.org/10.35377/saucis.04.01.879735

37. Rhanoui M, Mikram M, Yousfi S, Barzali S (2019) A CNN-BiLSTM Model for DocumentLevel Sentiment Analysis. Mach Learn Knowl Extr 1:832-847. https://doi.org/10.3390/make1030048

38. Deng Y, Wang L, Jia H, et al (2019) A Sequence-to-Sequence Deep Learning Architecture Based on Bidirectional GRU for Type Recognition and Time Location of Combined Power Quality Disturbance. IEEE Trans Ind Informatics 15:4481-4493. https://doi.org/10.1109/TII.2019.2895054

39. Moctezuma LA, Molinas M (2020) Towards a minimal EEG channel array for a biometric system using resting-state and a genetic algorithm for channel selection. Sci Rep 10:14917. https://doi.org/10.1038/s41598-020-72051-1

40. Ajami S, Mahnam A, Abootalebi V (2018) Development of a practical high frequency brain-computer interface based on steady-state visual evoked potentials using a single channel of EEG. Biocybern Biomed Eng 38:106-114. https://doi.org/10.1016/j.bbe.2017.10.004

41. Zarei A, Asl BM (2021) Automatic seizure detection using orthogonal matching pursuit, discrete wavelet transform, and entropy based features of EEG signals. Comput Biol Med 131:104250. https://doi.org/10.1016/j.compbiomed.2021.104250

42. Xu G, Ren T, Chen Y, Che W (2020) A One-Dimensional CNN-LSTM Model for Epileptic Seizure Recognition Using EEG Signal Analysis. Front Neurosci 14:. https://doi.org/10.3389/fnins.2020.578126

43. El-Fiqi H, Wang M, Salimi N, et al (2018) Convolution Neural Networks for Person 
Identification and Verification Using Steady State Visual Evoked Potential. In: 2018 IEEE International Conference on Systems, Man, and Cybernetics (SMC). IEEE, pp 1062-1069

44. Zhao H, Wang Y, Liu Z, et al (2019) Individual Identification Based on Code-Modulated Visual-Evoked Potentials. IEEE Trans Inf Forensics Secur 14:3206-3216. https://doi.org/10.1109/TIFS.2019.2912272

45. Wilaiprasitporn T, Ditthapron A, Matchaparn K, et al (2020) Affective EEG-Based Person Identification Using the Deep Learning Approach. IEEE Trans Cogn Dev Syst 12:486-496. https://doi.org/10.1109/TCDS.2019.2924648

46. Maiorana E (2020) Deep learning for EEG-based biometric recognition. Neurocomputing 410:374-386. https://doi.org/10.1016/j.neucom.2020.06.009

47. Chakladar D Das, Kumar P, Roy PP, et al (2021) A multimodal-Siamese Neural Network (mSNN) for person verification using signatures and EEG. Inf Fusion 71:17-27. https://doi.org/10.1016/j.inffus.2021.01.004

48. Rathi N, Singla R, Tiwari S (2021) Towards a role-based authentication system based on SSVEP-P300 hybrid brain-computer interfacing. Behav Inf Technol 1-17. https://doi.org/10.1080/0144929X.2021.1979655

49. Debie E, Moustafa N, Vasilakos A (2021) Session Invariant EEG Signatures using Elicitation Protocol Fusion and Convolutional Neural Network. IEEE Trans Dependable Secur Comput 1-1. https://doi.org/10.1109/TDSC.2021.3060775

50. Zhang Y, Li M, Shen H, Hu D (2021) A Permanency Investigation of SSVEP Signals in Brain Biometrics. In: 2021 IEEE 4th International Conference on Electronics Technology (ICET). IEEE, pp 758-762

51. Bassi PRAS, Rampazzo W, Attux R (2021) Transfer learning and SpecAugment applied to SSVEP based BCl classification. Biomed Signal Process Control 67:102542. https://doi.org/10.1016/j.bspc.2021.102542 